\title{
Interference alignment using finite and dependent channel extensions: the single beam case *
}

\author{
Ruoyu Sun $^{\dagger}$ and Zhi-Quan Luo ${ }^{\ddagger}$
}

\begin{abstract}
Vector space interference alignment (IA) is known to achieve high degrees of freedom (DoF) with infinite independent channel extensions, but its performance is largely unknown for a finite number of possibly dependent channel extensions. In this paper, we consider a $K$-user $M_{t} \times M_{r}$ MIMO interference channel (IC) with arbitrary number of channel extensions $T$ and arbitrary channel diversity order $L$ (i.e., each channel matrix is a generic linear combination of $L$ fixed basis matrices). We study the maximum DoF achievable via vector space IA in the single beam case (i.e. each user sends one data stream). We prove that the total number of users $K$ that can communicate interference-free using linear transceivers is upper bounded by $N L+N^{2} / 4$, where $N=\min \left\{M_{t} T, M_{r} T\right\}$. An immediate consequence of this upper bound is that for a SISO IC the DoF in the single beam case is no more than $\min \left\{\sqrt{\frac{5}{4} K}, L+\frac{1}{4} T\right\}$. When the channel extensions are independent, i.e. $L$ achieves the maximum $M_{r} M_{t} T$, we show that this maximum DoF lies in $\left[M_{r}+M_{t}-1, M_{r}+M_{t}\right]$ regardless of $T$. Unlike the well-studied constant MIMO IC case, the main difficulty is how to deal with a hybrid system of equations (zero-forcing condition) and inequalities (full rank condition). Our approach combines algebraic tools that deal with equations with an induction analysis that indirectly considers the inequalities.
\end{abstract}

\section{Introduction}

Interference alignment (IA) has recently attracted considerable attention due to its significant potential to achieve high throughput in wireless networks. However, many current IA schemes rely on two rather unrealistic assumptions: an exponentially (in the number of users) many independent channel extensions and perfect channel state information at the transmitters (CSIT). In this paper, we study IA schemes with the first assumption relaxed (while still assuming perfect CSIT). In particular, we

\footnotetext{
*This work is supported in part by the National Science Foundation, grant number TF-0728676.

${ }^{\dagger} \mathrm{R}$. Sun is with the Department of Electrical and Computer Engineering, University of Minnesota, Minneapolis, MN 55455. Email: sunxx394@umn.edu.

${ }^{\ddagger}$ Z.-Q. Luo is with the Chinese University of Hong Kong, Shenzhen, China. He is also affiliated with the Department of Electrical and Computer Engineering, University of Minnesota, Minneapolis, MN 55455. Email: luozq@ cuhk.edu.cn.
} 
consider the following problem: with a finite number of possibly dependent channel extensions, what is the performance limit of any IA scheme?

\subsection{Prior Work}

Introduced in [1] for MIMO X channels and in [2] for interference channels, interference alignment has been shown to be able to achieve high Degrees of Freedom (DoF). Roughly speaking, the DoF is the first-order approximation of the network capacity in the high SNR regime and can be interpreted as the number of data streams that can be transmitted in an interference-free manner. For a $K$-user timevarying or frequency selective SISO interference channel (IC), Cadambe and Jafar [2] constructed an asymptotic IA scheme (referred herein as C-J scheme) that achieves $K / 2 \mathrm{DoF}$, provided that the number

of independent channel extensions grows exponentially in $K^{2}$. This surprising result matches the outer bound proven by Host-Madsen and Nosratinia [3], and implies that each user can get "half-the-cake", with the cake representing the maximum achievable DoF in a point-to-point channel, regardless of the number of interfering users present in the system. This result can be easily extended to a MIMO interference channel where each transmitter/receiver has the same number of antennas [2].

The C-J scheme in [2] belongs to the class of vector space IA strategies that apply linear transceivers and align the interference subspaces at each receiver into a low dimensional space. This is in contrast to signal level IA schemes which apply lattice codes at transmitters with the goal of aligning the codes of interference at each receiver into few lattice points. Although signal level IA schemes can theoretically achieve a higher DoF than vector space IA schemes (e.g., for constant SISO interference channel [4] and constant MIMO interference channel [5]), they require unrealistic assumptions of infinite precision of the channel state information and exponentially (in terms of the number of users) large codebooks. In this paper, we focus on vector space IA schemes and study the maximum DoF they can achieve.

For a MIMO IC with no channel extension (which is an extreme case of finite extensions), the references [6-8] have analyzed the achievable DoF using algebraic techniques. In particular, the authors of [6] formulated the IA condition as a polynomial system of equations with beamforming vectors being the variables, and defined the notion of a "proper" system for which the number of equations is no more than the number of variables in every subsystem of equations. For the single beam case (i.e. every user transmits a single data stream), they applied the Bernstein's theorem to show that the IA condition is feasible only if it is proper, thus obtaining a DoF upper bound by counting the number of equations and number of variables (referred herein as the dimensionality counting argument). More recently, Razaviyayn et al. [7] and Bresler et al. [8] independently established the same DoF upper bound (based on the counting argument) without the restriction of one data stream per user. For the special case that each user transmits $d$ data streams in a $M \times M$ MIMO channel with no channel extension, their result implies that the DoF is upper bounded by $2 M$ (more precisely, $2 M K /(K+1)$ ). Compared to the $M$ DoF that can be achieved by simply using orthogonalizing strategies, the gain brought about by interference alignment, called "alignment gain" in [8], is upper bounded by 2 . This is significantly smaller than the 
$K / 2$ alignment gain for a $K$-user $M \times M$ MIMO interference channel with $O\left(e^{K^{2}}\right)$ independent channel extensions [2]. The achievability of the DoF upper bound has been established for the case $d_{k}=d, \forall k$ and $M_{k}, N_{k}$ divisible by $d$ in [7], and for the case $M_{k}=N_{k}=N, d_{k}=d, \forall k$ in [8], where $d_{k}$ is the number of data streams user $k$ transmits and $M_{k}, N_{k}$ are the number of antennas at transmitter $k$ and receiver $k$ respectively. The issue of how to design linear transceivers that can achieve interference alignment has been considered in [9].

For the case of an arbitrary number of independent time or frequency extensions, the maximum DoF has been characterized only for the case $K=3[2,10]$. In particular, for a 3-user $M \times M$ MIMO interference channel, reference [2] showed that the maximum DoF is $3 M / 2$, either with an even $M$ and no channel extension or with an odd $M$ and 2 channel extensions. Reference [10] has further considered a 3-user SISO interference channel with $L$ independent channel extensions for a general $L$, and characterized the maximum DoF as a function of $L$. However, it is not clear how to extend these results to general $K$.

\subsection{Contributions of This Work}

\subsubsection{Overview of Contributions}

The existing IA results for the $K$-user IC can be roughly divided into two categories: "optimistic" results that achieve $O(K)$ alignment gain [2,4,5], and "pessimistic" results that bound the alignment gain by 2 [6-8]. The optimistic results require impractical assumptions of exponentially many independent channel extensions, while the pessimistic results only apply to a restricted scenario with no channel extensions. Then a problem of great interest is how much alignment gain (or DoF) can be achieved

for a practical model with polynomially many and possibly dependent channel extensions. Little was known before about this problem: it was not even known whether the trivial DoF upper bound $K / 2$ can be improved or not. The existing works [8] and [11] slightly improved the lower bound in two scenarios from 1 to a number less than 3/2 (they proved achievability of certain DoF values for 3-user SISO IC, which also imply the achievability of these DoF values for $K$-user SISO IC). Still there is a huge gap between $3 / 2$ and the upper bound $K / 2$.

To model the possible dependency among channel extensions, we introduce the notion of channel diversity order. Specifically, we say an interference channel has a diversity order $L$ if each channel matrix is a generic linear combination of $L$ fixed basis matrices. The benefit of introducing this notion is that many seemingly different practical channel models, such as the $L$-tap SISO IC, the SISO/MIMO IC with block-independent time or frequency extensions and the SISO/MIMO IC with asymmetric complex signalling, can be treated within this unified framework. More examples with diversity order $L$ will be given later.

Our ultimate goal is to characterize the maximum DoF (achievable via vector space IA) of a $K$-user MIMO IC with any number of channel extensions and any channel diversity order. As an intermediate 
step, in this work we restrict to the single beam case, i.e. each user sends $d=1$ data stream to its intended receiver. Under this restriction, the DoF $K d / T$ (total DoF per channel use) becomes $K / T$, thus bounding the DoF amounts to bounding the total number of users $K$ for a given $T$. Note that the number of users that can be accommodated to achieve a given QoS (Quality of Service) threshold is itself an interesting performance metric for a wireless system. Our results characterize this metric in the IA context: given $T$ channel extensions (and any $L$ ) and the QoS requirement of 1 aggregate DoF per-user (i.e. 1/T DoF per-channel-use per-user), how many users can simultaneously communicate in an interference-free manner? We emphasize that no upper bound for the number of supported users with general $T$ was known before (the trivial DoF upper bound $K / 2$ does not translate to any bound on $K$ ), and our results provide the first nontrivial upper bound $K \leq O\left(T^{2}\right)$ for any $T$ and any $L$.

The bound on $K$ can be immediately translated to the bound on the DoF under the single-beam restriction. Roughly speaking, our results imply that in the single-beam case, the alignment gain (approximately equal to the DoF divided by the number of antennas per transmitter/receiver) is upper bounded by $O(\sqrt{K})$, regardless of the diversity order or the number of channel extensions. This upper bound is much smaller than the trivial upper bound $K / 2$. For the special case of independent channel extensions (i.e. the diversity order achieves the maximum), we establish a stronger upper bound of 2 (Theorem 3.1). Therefore, under the single-beam restriction we can not expect an alignment gain as high as $K / 2$ (or even $K^{1 / 2+\epsilon}$ where $\epsilon>0$ ). An interesting open question is whether the $O(\sqrt{K})$ DoF can be achieved in the single-beam case with finite diversity order.

\subsubsection{Detailed Summary of Contributions}

We consider a general MIMO IC with an arbitrary number of channel extensions and an arbitrary channel diversity order, and prove upper bounds on the DoF (achievable via vector space IA) in the single beam case, as well as the upper bounds on the number of users that can transmit interferencefree. Our main contributions, both theoretical and technical, are summarized as follows.

(i) For a general diversity order $L$, we establish a universal DoF upper bound, which is the first such bound for IA with dependent channel extensions. Specifically, for a $K$-user $M_{t} \times M_{r}$ MIMO IC with $T$ channel extensions and channel diversity order $L$, we prove that the total number of users $K$ that can communicate interference-free using linear transceivers is upper bounded by $N L+N^{2} / 4$, where $N=\min \left\{M_{r} T, M_{t} T\right\}$. We emphasize that our result applies to any number of users, any channel diversity order and any number of channel extensions. This result immediately leads to DoF upper bounds of many practical channels in the single beam case. For instance, for a $K$-user SISO IC in the

single beam case, the maximum DoF is no more than $\min \left\{\sqrt{\frac{5}{4} K}, L+\frac{1}{4} T\right\}$, where $T$ is the number of channel extensions (not necessarily independent extensions).

(ii) For the extreme case that the channel extensions are independent (i.e. maximum channel diversity order), we prove a constant DoF upper bound. Specifically, for a $M_{t} \times M_{r}$ MIMO IC with 
$T$ independent channel extensions in the single beam case, we show that the maximum DoF lies in $\left[M_{r}+M_{t}-1, M_{r}+M_{t}\right]$ regardless of $T$. The same DoF upper bound has been obtained in [12] for SISO IC and [13] for MIMO IC; however, their results require a strong assumption on the beamformers. Our result generalizes the previous DoF bound of MIMO IC with no channel extension in the single beam case [6]. Compared to the $K / 2 \mathrm{DoF}$ achievable for the multi-beam case [2], our result shows that with the single-stream restriction the performance gain provided by vector space IA is very limited.

(iii) Our main technical contribution is to develop an induction analysis framework that, combined with algebraic tools, can determine the feasibility of a hybrid system of equations and inequalities. We believe this framework can be of use in other IA contexts. In particular, it is now well-known that the feasibility of an IA system is equivalent to the solvability of a system of polynomial equations when the direct link channel matrix is generic [6-8]. We emphasize that this equivalence no longer holds if the direct link matrices are not generic; in fact, in the latter case, the problem is reduced to the feasibility of a hybrid system of equations and inequalities. Since algebraic geometry tools usually cannot deal with hybrid systems directly, other techniques are needed. We develop an induction analysis that leverages the recursive structure of the IA system, while indirectly considers the inequalities. To deal with the equations, we generalize algebraic tools used in the existing IA literature, which may be of independent interest. Finally, we also provide some clarifications on the use of Bernstein's theorem in the IA context (more specifically, the so called counting argument to determine the feasibility of a system of polynomial equations).

\section{System Model}

\subsection{Channel Diversity Order}

To model the possible dependency among channel extensions, we introduce the notion of channel diversity order. The benefit of introducing this notion is that many seemingly different practical channel models can be treated within this unified framework.

We say a $K$-user symmetric interference channel has a diversity order $L$ if each channel matrix $H_{i j}, 1 \leq i, j \leq K$ is a linear combination of $L$ fixed matrices $A_{1}, \ldots, A_{L} \in \mathbb{C}^{N_{r} \times N_{t}}: H_{i j}=\tau_{i j}^{1} A_{1}+\cdots+$ $\tau_{i j}^{L} A_{L}$, where $A_{1}, A_{2}, \ldots, A_{L}$ are linearly independent. We call $A_{1}, \ldots, A_{L}$ the building blocks of this interference channel. For a symmetric interference channel with $T$ time or frequency extensions where each transmitter (receiver) is equipped with $M_{t}\left(M_{r}\right)$ antennas, the dimensions of the channel matrices $N_{t}, N_{r}$ are related to $T, M_{t}, M_{r}$ through the relations $N_{t}=M_{t} T, N_{r}=M_{r} T$.

We are interested in the case that the coefficients $\tau_{i j}^{l}, 1 \leq i, j \leq K, 1 \leq l \leq L$ are generic (e.g. independently drawn from the same or different continuous random distributions). Strictly speaking, a property is said to hold for generic $x=\left(x_{1}, \ldots, x_{n}\right) \in \mathbb{C}^{n}$ if there is a nonzero polynomial $f$ such that the property holds in set $\{x \mid f(x) \neq 0\}$. In the so called Zariski topology, such a set $\{x \mid f(x) \neq 0\}$ is 
called a Zariski open set. In other words, a property holds generically if it holds over a Zariski open set (this implies that it holds with probability one if $x_{i}$ 's are drawn from continuous random distributions).

In order to avoid degenerate channel matrices, we impose some mild requirements on the building blocks $A_{1}, \ldots, A_{L}$. Define

$$
\begin{aligned}
\Psi=\left\{\left(A_{1}, \ldots, A_{L}\right) \mid\right. & A_{\ell} \in \mathbb{C}^{N_{r} \times N_{t}}, \ell=1, \ldots, L ; \\
& \left.\exists k_{1}, \ldots, k_{L} \in \mathbb{C}, \text { s.t. } \operatorname{rank}\left(k_{1} A_{1}+k_{2} A_{2}+\cdots+k_{L} A_{L}\right)=\min \left\{N_{r}, N_{t}\right\}\right\} .
\end{aligned}
$$

Then for any $\left(A_{1}, \ldots, A_{L}\right) \in \Psi$, each channel matrix $H_{i j}=\tau_{i j}^{1} A_{1}+\cdots+\tau_{i j}^{L} A_{L}$ is full rank for generic $\left(\tau_{i j}^{l}\right)_{1 \leq i, j \leq K, 1 \leq l \leq L}$.

We describe below several channel models with finite diversity order.

Example 2.1: A SISO IC with $L$ generic time or frequency extensions has a diversity order $L$. Indeed, each channel matrix $H_{i j}=\operatorname{diag}\left(H_{i j}^{(1)}, \ldots, H_{i j}^{(L)}\right)$ is a generic linear combination of $L$ diagonal matrices $\operatorname{diag}(1,0, \ldots, 0), \operatorname{diag}(0,1, \ldots, 0), \ldots, \operatorname{diag}(0,0, \ldots, 1)$, which serve as the building blocks. Such a channel model is commonly used in the interference alignment studies $[2,10]$.

Example 2.2: A SISO IC with $L$-tap discrete channels and $N \geq L$ frequency extensions has a diversity order $L$. Indeed, suppose the channel between all transmitter-receiver pairs $(k, j)$ is an $L$ tap channel with the same time delays $\lambda_{1}<\lambda_{2}<\cdots<\lambda_{L}$, where $\lambda_{\ell} \in\{0,1, \ldots, N-1\}$. The channel matrix (in the frequency domain) $H_{i j}$ is an $N \times N$ diagonal matrix, and can be expressed as $H_{i j}=\sum_{l=1}^{L} \tau_{i j}^{l} A_{l}$, where $A_{l}=\operatorname{diag}\left(1, e^{\frac{2 \pi \lambda_{l}}{N} \sqrt{-1}}, \ldots, e^{\frac{2(N-1) \pi \lambda_{l}}{N} \sqrt{-1}}\right)$, and $\tau_{i j}^{l}$ is the $l^{\prime}$ th tap channel coefficient between transmitter $j$ and receiver $i$.

Example 2.3: A SISO IC with $L$ blocks of generic channel extensions has a diversity order $L$. More specifically, suppose there are $N$ channel extensions that can be divided into $L$ blocks, where the channel coefficients in the same block are equal, and are independent across blocks. This is the well-known block-fading channel model. As a simple example, suppose $N_{1}+N_{2}$ channel extensions are divided into two blocks with $N_{1}, N_{2}$ channel extensions respectively and the channel matrices can be expressed as $H_{i j}=\operatorname{diag}\left(\tau_{i j}^{1} I_{N_{1}}, \tau_{i j}^{2} I_{N_{2}}\right)$, then the building blocks are $A_{1}=\operatorname{diag}\left(I_{N_{1}}, 0\right), A_{2}=\operatorname{diag}\left(0, I_{N_{2}}\right)$. In general, the building blocks are block diagonal matrices consisting of identity matrices and zero matrices. Such a channel model considers a more general "coherence block structure" than the model with constant extensions or generic extensions. Special asymmetric coherence block structures have been considered in blind IA $[14,15]$ to achieve high DoF. For a general symmetric coherence block structure, the DoF has not been studied in the literature yet.

Example 2.4a: A constant MIMO IC ("constant” means flat-fading channel and no channel extension) where each transmitter has $M_{t}$ antennas and each receiver has $M_{r}$ antennas (we call $M_{t} \times M_{r}$ MIMO IC) has a diversity order $M_{t} M_{r}$. The channel matrix $H_{i j}$ is a $M_{r} \times M_{t}$ matrix with generic entries. The building blocks are $E_{k l}, 1 \leq k \leq M_{r}, 1 \leq l \leq M_{t}$, where $E_{k l}$ denotes the matrix with only one nonzero entry 1 in the entry $(k, l)$. Such a channel model has been studied in references $[7,8]$. 
Example 2.4b: An $M_{t} \times M_{r}$ MIMO IC with $T$ constant channel extensions has a diversity or$\operatorname{der} M_{t} M_{r}$. The channel matrix $H_{i j}$ is a $T M_{r} \times T M_{t}$ block diagonal matrix $\left[\begin{array}{cccc}\bar{H}_{i j} & 0 & \cdots & 0 \\ 0 & \bar{H}_{i j} & \cdots & 0 \\ \vdots & \vdots & \ddots & \vdots \\ 0 & 0 & \cdots & \bar{H}_{i j}\end{array}\right]$, where $\bar{H}_{i j}$ is a $M_{r} \times M_{t}$ matrix with generic entries. The building blocks are block diagonal matri$\operatorname{ces}\left[\begin{array}{cccc}E_{k \ell} & 0 & \cdots & 0 \\ 0 & E_{k \ell} & \cdots & 0 \\ \vdots & \vdots & \ddots & \vdots \\ 0 \cdots & 0 & \cdots & E_{k \ell}\end{array}\right]$. To our knowledge, such a channel model has not been considered in the interference alignment area.

Example 2.4c: An $M_{t} \times M_{r}$ MIMO IC with $T$ generic channel extensions has a diversity order $M_{t} M_{r} T$. The channel matrix $H_{i j}$ is a $M_{r} T \times M_{t} T$ block diagonal matrix $\left[\begin{array}{cccc}H_{i j}^{1} & 0 & \cdots & 0 \\ 0 & H_{i j}^{2} & \cdots & 0 \\ \vdots & \vdots & \ddots & \vdots \\ 0 & 0 & \cdots & H_{i j}^{T}\end{array}\right]$, where each block $H_{i j}^{l}$ is a $M_{r} \times M_{t}$ matrix with generic entries. Such a channel model is a generalization of the SISO channel in Example 2.1, and has been investigated in $[5,16]$ for the case $T \rightarrow \infty$.

At last, we show an example where asymmetric complex signaling (ACS) [11] can double the channel diversity order.

Example 2.5: Using ACS, a SISO IC with any number of constant channel extensions has a diversity order 2. The idea of ACS is to convert each complex channel to two real channels, thus doubling the ambient dimension and the diversity order. In a point-to-point channel with input $x \in \mathbb{C}$, channel $h \in \mathbb{C}$, noise $n \in \mathbb{C}$ and output $y \in \mathbb{C}$, we have

$$
y=h x+n \Longleftrightarrow\left(\begin{array}{c}
\operatorname{Re}(y) \\
\operatorname{Im}(y)
\end{array}\right)=\left(\begin{array}{cc}
\operatorname{Re}(h) & -\operatorname{Im}(h) \\
\operatorname{Im}(h) & \operatorname{Re}(h)
\end{array}\right)\left(\begin{array}{c}
\operatorname{Re}(x) \\
\operatorname{Im}(x)
\end{array}\right)+\left(\begin{array}{c}
\operatorname{Re}(n) \\
\operatorname{Im}(n)
\end{array}\right),
$$

where $\operatorname{Re}(\cdot), \operatorname{Im}(\cdot)$ denote the real part and the imaginary part of a complex number respectively. In a $K$-user SISO IC with $T$ constant channel extensions, suppose the channel matrix between transmitter $j$ and receiver $k$ is a $T \times T$ diagonal matrix $\operatorname{diag}\left(h_{k j}, \ldots, h_{k j}\right)$. The channel diversity order is 1 , with the identity matrix $I$ being the building block. Using ACS, the channel matrix becomes a $2 T \times 2 T$ block diagonal matrix

$$
H_{k j}=\left[\begin{array}{ccccc}
\operatorname{Re}\left(h_{k j}\right) & -\operatorname{Im}\left(h_{k j}\right) & \cdots & 0 & 0 \\
\operatorname{Im}\left(h_{k j}\right) & \operatorname{Re}\left(h_{k j}\right) & \cdots & 0 & 0 \\
\vdots & \vdots & \ddots & \vdots & \vdots \\
0 & 0 \ldots & \cdots & \operatorname{Re}\left(h_{k j}\right) & -\operatorname{Im}\left(h_{k j}\right) \\
0 & 0 \ldots & \cdots & \operatorname{Im}\left(h_{k j}\right) & \operatorname{Re}\left(h_{k j}\right)
\end{array}\right]
$$


The two basis matrices (or building blocks) are

$$
I=\left[\begin{array}{ccccc}
1 & 0 & \cdots & 0 & 0 \\
0 & 1 & \cdots & 0 & 0 \\
\vdots & \vdots & \ddots & \vdots & \vdots \\
0 & 0 \ldots & \cdots & 1 & 0 \\
0 & 0 \ldots & \cdots & 0 & 1
\end{array}\right] \text { and }\left[\begin{array}{ccccc}
0 & -1 & \cdots & 0 & 0 \\
1 & 0 & \cdots & 0 & 0 \\
\vdots & \vdots & \ddots & \vdots & \vdots \\
0 & 0 \ldots & \cdots & 0 & -1 \\
0 & 0 \ldots & \cdots & 1 & 0
\end{array}\right]
$$

This gives a diversity order of $L=2$.

We remark that the notion of channel diversity order we define here is different from the number of channel extensions. Although in Example 2.1 with generic channel extensions, channel diversity order coincides with the number of channel extensions, in general this is not the case. For instance, the networks in Example 2.4a and Example 2.4b have the same diversity order, but different numbers of channel extensions. In Example 2.3, the number of channel extensions is larger than the channel diversity order. In general, channel diversity is a more limited resource than the number of channel extensions.

\subsection{System Model and IA Condition}

Consider a $K$-user interference channel with diversity order $L$, i.e. each channel matrix $H_{i j}$ is the linear combination of $L$ fixed matrices $A_{1}, \ldots, A_{L} \in \mathbb{C}^{N_{r} \times N_{t}}$, where $A_{1}, \ldots, A_{L} \in \Psi$ as defined in (1).

In this work, we focus on vector space interference alignment strategies which deploy linear transmit and receive beamformers and align the subspaces of interference into a low dimensional space at each receiver. Specifically, suppose transmitter $k$ intends to transmit $d_{k}$ independent data streams $s_{k} \in \mathbb{C}^{d_{k} \times 1}$ to receiver $k$. In the transmitter side, a linear beamforming matrix $V_{k} \in \mathbb{C}^{N_{t} \times d_{k}}$ is used to encode $s_{k}$, i.e. transmitter $k$ sends a signal $x_{k}=V_{k} s_{k}$. Receiver $k$ receives a signal

$$
y_{k}=H_{k k} x_{k}+\sum_{j \neq k} H_{k j} x_{j}+n_{k}=H_{k k} V_{k} s_{k}+\sum_{j \neq k} H_{k j} V_{j} s_{j}+n_{k},
$$

where $n_{k} \in C^{N_{r} \times 1}$ is a zero mean additive Gaussian white noise. The receiver $k$ applies a receive beamforming matrix $U_{k} \in \mathbb{C}^{N_{r} \times d_{k}}$ to process the received signal to obtain an estimate of the signal

$$
\hat{s}_{k}=U_{k}^{H} y_{k}=U_{k}^{H} H_{k k} V_{k} s_{k}+\sum_{j \neq k} U_{k}^{H} H_{k j} V_{j} s_{j}+U_{k}^{H} n_{k} .
$$

In vector space interference alignment, we want to design beamforming matrices $\left\{V_{k}\right\}$ such that all interference is aligned into a small space that is linearly independent of the signal space, thus the interference can be eliminated by multiplying zero-forcing matrices $\left\{U_{k}\right\}$. More formally, $\left\{V_{k}, U_{k}\right\}$ must satisfy the following IA condition $[6-8,17]$ :

$$
\begin{aligned}
& U_{k}^{H} H_{k j} V_{j}=0, \quad \forall 1 \leq k \neq j \leq K, \\
& \operatorname{rank}\left(U_{k}^{H} H_{k k} V_{k}\right)=d_{k}, \quad \forall 1 \leq k \leq K .
\end{aligned}
$$


The first equation implies that the interference from any user can be eliminated at the receiver side by using the zero-forcing beamforming matrix $U_{k}$. The second equation ensures that no information in $s_{k}$ is lost when multiplied by $U_{k}^{H} H_{k k} V_{k}$.

We say that a DoF $\bar{d}$ is achievable by a vector space IA scheme if there exist $V_{k} \in \mathbb{C}^{N_{t} \times d_{k}}, U_{k} \in$ $\mathbb{C}^{N_{r} \times d_{k}}, k=1, \ldots, K$, such that (3) holds and $\bar{d}=\left(d_{1}+\cdots+d_{K}\right) / T$, where $T$ is the number of channel uses. Throughout this paper, we only consider the total DoF (or DoF for short) that is achievable by a vector space IA scheme.

\section{Main Results}

Consider a symmetric interference channel with a given diversity order. In this section, we present several DoF bounds for interference channels in which each user transmits a single data stream (i.e. singlebeam case, $d_{k}=1, \forall k$ ), as well as the bounds on the number of users that can transmit interference-free using linear transceivers.

The first result shows that in a $M_{t} \times M_{r}$ MIMO IC with generic channel extensions (in this case the channel diversity order achieves the maximum), the DoF is upper bounded by $M_{r}+M_{t}$ in the single beam case. As a special case, the DoF of a SISO IC in the single beam case is upper bounded by 2 , regardless of the number of generic channel extensions. The proof of this result will be provided in Section 5 .

Theorem 3.1 Consider a $K$-user $M_{t} \times M_{r}$ MIMO interference channel with $T$ independent channel extensions (all the channel coefficients are independently drawn from continuous random distributions). Suppose each user transmits single data stream. Then the following results hold with probability one.

(a) If a vector space IA scheme exists, then

$$
K \leq\left(M_{r}+M_{t}\right) T-1
$$

(b) If $K=\left(M_{r}+M_{t}-1\right) T$, then a vector space IA scheme exists.

(c) The maximum DoF achievable (via vector space IA) in this single beam case is no more than $M_{r}+M_{t}$, and no less than $M_{r}+M_{t}-1$.

Remark 1: The bound (4) can be slightly improved to $K \leq\left(M_{r}+M_{t}\right) T-2$ if $T \geq 2$, by using the techniques of $[12,13]$. Since we are mainly interested in the asymptotic DoF bound in this work (as discussed in Section 1.2), we choose to just present (4).

Remark 2: By a variant of our proof, it is possible to show a slightly stronger result that the same properties in fact hold for generic channel coefficients (i.e. for all channel coefficients in a Zariski open set). Similarly, Theorem 3.2 can be shown to hold for "generic" coefficients. 
Notice that part (c) of Theorem 3.1 is a direct consequence of part (a) and part (b) since for the single-beam case the DoF equals $K / T$. Part (b) of Theorem 3.1 can be proved by the result in [7]. In fact, [7, Theorem 2] implies that when $T=1$ and $K=M_{r}+M_{t}-1$, the DoF tuple $(1, \ldots, 1)$ is achievable (since the number of transmit/receive antennas are divisible by $d=1$ ). For general $T$, we can use an orthogonalizing scheme to achieve $(1, \ldots, 1)$ for $K=\left(M_{r}+M_{t}-1\right) T$ users: for each of the $T$ channel uses, $\left(M_{r}+M_{t}-1\right)$ users transmit a single data stream interference-free while other users do not transmit; in total, $K=\left(M_{r}+M_{t}-1\right) T$ users can transmit a single data stream interference-free. It remains to prove part (a) of Theorem 3.1 and Section 5 is devoted to such a proof.

Part (a) of Theorem 3.1, although seems simple, is not easy to prove. It has been studied in [12] for SISO IC and [13] for MIMO IC under strong assumptions (e.g., the beamforming solutions are independent and marginally isotropic). Essentially their results require the assumption that the beamforming solutions do not have any zero entries, which is an artificial assumption being added so that the proof in [6] can be directly applied. However, as shown later, the difficulty of proving part (a) is exactly due to the possibility that the beamforming solutions may contain zero entries. The new technique developed herein to tackle this difficulty is one of the main contributions of this paper. It is also crucial to prove other results of this paper.

Theorem 3.1 states that for the single-beam case, the maximum DoF lies in the region $\left[M_{r}+M_{t}-\right.$ $\left.1, M_{r}+M_{t}\right]$ for any $T$. For the special case $T=1$ (i.e. constant MIMO IC), this result has been proven in [7,8], and [7] shows that the maximal DoF is indeed $M_{r}+M_{t}-1$. Therefore, for the single-beam case, increasing the number of generic channel extensions does not lead to a significant improvement of the DoF.

When $M_{t}=M_{r}=M$, Theorem 3.1 implies that the DoF is no more than $2 M$ for the single-beam case. This result is in sharp contrast to $\frac{K}{2} M$ DoF in a $M \times M$ MIMO IC when each user is allowed to transmit multiple data streams [2]. Therefore, the single data stream restriction appears to be a throughput limiting factor even in the presence of an arbitrary number of generic channel extensions. It would be interesting to find how the DoF scales with the number of data streams that each user can transmit.

The second result considers a general channel model with any channel diversity order and any number of users, antennas and channel extensions. We provide a universal upper bound on the number of users that can be accommodated to achieve IA. This upper bound is a function of the diversity order $L$ and the minimum of the transmit dimension and the receive dimension $\min \left\{N_{t}, N_{r}\right\}$. The proof of this result will be presented in Section 6.

Theorem 3.2 Consider a $K$-user $M_{t} \times M_{r}$ MIMO interference channel with $T$ channel extensions (possibly dependent extensions). Let $N_{t}=M_{t} T, N_{r}=M_{r} T$ and

$$
N=\min \left\{N_{t}, N_{r}\right\}
$$

Suppose the channel diversity order is $L$, i.e. the channel matrices $H_{i j}=\tau_{i j}^{1} A_{1}+\cdots+\tau_{i j}^{L} A_{L} \in \mathbb{C}^{N_{r} \times N_{t}}$, 
with $\left(A_{1}, \ldots, A_{L}\right) \in \Psi$ defined in (1). Then for almost all coefficients $\left(\tau_{i j}^{l}\right)_{1 \leq i, j \leq K, 1 \leq l \leq L}$, the DoF tuple $\left(d_{1}, \ldots, d_{K}\right)=(1,1, \ldots, 1)$ is achievable via a vector space IA scheme only if

$$
K \leq N L+\frac{N^{2}}{4}
$$

Theorem 3.2 gives an upper bound on $K_{\max }$, where $K_{\max }$ is the maximal number of users that can be accommodated to achieve IA for almost all coefficients $\left(\tau_{i j}^{l}\right)$. Note that it was not even known before whether an upper bound on $K_{\max }$ exists; in other words, we have improved the bound of $K_{\max }$ from $K_{\max } \leq \infty$ to $K \leq N L+\frac{N^{2}}{4}$.

How tight is our bound (5)? A trivial lower bound that can be achieved by any channel model is $K_{\max } \geq N$, simply using orthogonizing schemes. Orthogonizing schemes can achieve better lower bound

$$
K_{\max } \geq N L
$$

for some channel models, such as $1 \times M_{r}$ SIMO IC with $T$ constant channel extensions $\left(N_{t}=T, N_{r}=\right.$ $T M_{r}$ and $N=T, L=M_{r}$ for this setting). For this channel model, when $T=M_{r}$, i.e. the number of channel extensions equals the number of receive antennas per-user, the lower bound $N L=M_{r}^{2}$ is of the same order as the upper bound $N L+\frac{N^{2}}{4}=\frac{5}{4} M_{r}^{2}$. In other words, there exists a very special case in which the bound (5) is almost optimal. The bound (5) can be very loose in some cases, such as the SISO IC with indepedent channel extensions. In this case Theorem 3.1 establishes a bound $K \leq 2 T-1$, while the bound (5) $K \leq 2 T+\frac{T^{2}}{4}$ is much looser. In general, there is a gap of $N^{2} / 4$ between the bound (5) and the lower bound for a special case (6) (for general channel models whereby only a lower bound $K_{\max } \geq N$ is known, the gap increases to $\left.N^{2} / 4+(L-1) N\right)$. Nevertheless, since the lower bound is only achieved by simple orthogonizing schemes, it is possible that more sophisticated IA schemes can achieve better lower bounds. Further evaluation of the tightness of our bound (5) is left as future work.

Theorem 3.2 can be used to derive DoF upper bounds of various channel models for the singlebeam case. We first consider the SISO IC, and provide two upper bounds: one in terms of $K$ and another in terms of $T$ and $L$.

Corollary 3.1 Consider a K-user SISO interference channel with a diversity order $L$ and $T$ channel extensions. If $d_{k}=1, \forall k$, then the maximum DoF achievable via a vector space IA scheme is at most $\min \left\{\sqrt{\frac{5}{4} K}, L+\frac{1}{4} T\right\}$

Proof of Corollary 3.1: In the SISO IC, the transmit dimension $N_{t}$ and receive dimension $N_{r}$ are both equal to $T$, thus $N=\min \left\{N_{t}, N_{r}\right\}=T$. According to Theorem 3.2, we have

$$
K \leq T L+\frac{T^{2}}{4}
$$

thus the DoF for the single-beam case $\mathrm{DoF}_{\mathrm{s}}$ can be bounded as

$$
\mathrm{DoF}_{\mathrm{s}}=\frac{K d}{T}=\frac{K}{T} \leq L+\frac{1}{4} T \text {. }
$$


Since the channel matrices are $T \times T$ diagonal matrices, the diversity order $L$ should be no more than $T$. Then (7) implies

$$
K \leq T^{2}+\frac{T^{2}}{4}=\frac{5}{4} T^{2} .
$$

Thus the DoF for the single-beam case

$$
\operatorname{DoF}_{\mathrm{s}}=\frac{K}{T} \leq \sqrt{\frac{5}{4} K}
$$

\section{Q.E.D.}

Corollary 3.1 applies to Example 2.1-2.3 in Section 2.1. Specifically, the achievable DoF is upper bounded by $\min \left\{\sqrt{\frac{5}{4} K}, L+\frac{1}{4} T\right\}$ for the single-beam case in the following networks: the SISO IC with $T=L$ generic time or frequency extensions (Example 2.1), $L$-tap SISO IC with $T$ frequency extensions (Example 2.2), the SISO IC with $L$ generic blocks of $T$ channel extensions (Example 2.3). For Example 2.1, this bound is weaker than the bound of 2 provided by Theorem 3.1. Nevertheless, for Example 2.2 and Example 2.3, this is the first general DoF upper bound to our knowledge.

Compared with the $K / 2$ achievable DoF for a SISO IC in the multi-beam case, Corollary 3.1 proves a $O(\sqrt{K})$ upper bound in a SISO IC for the single-beam case, regardless of the channel diversity order or the number of channel extensions. Corollary 3.1 also shows that the DoF for the single-beam case is bounded by $L+\frac{1}{4} T$, which increases linearly both with $L$ and $T$. When the channel extensions are not generic, it is not known whether the $O(\sqrt{K})$ bound or the $L+\frac{1}{4} T$ bound can be improved to a constant bound.

Applying Theorem 3.2 to the MIMO IC also obtains a $O(\sqrt{K})$ DoF bound in the single beam case. Specifically, for an $M_{t} \times M_{r}$ MIMO IC with $T$ possibly dependent channel extensions, the channel diversity order $L \leq M_{r} M_{t} T$. Assume $M_{t} \leq M_{r}$, then $N=M_{t} T$, and (5) becomes

$$
K \leq N L+\frac{N^{2}}{4}=M_{t}^{2} M_{r} T^{2}+\frac{1}{4} M_{t}^{2} T^{2} \leq \frac{5}{4} M_{t}^{2} M_{r} T^{2},
$$

which further implies that the DoF in the single-beam case $\mathrm{DoF}_{\mathrm{s}}$ can be bounded as

$$
\mathrm{DoF}_{\mathrm{s}}=\frac{K}{T} \leq M_{t} \sqrt{\frac{5}{4} M_{r} K}
$$

This bound is possibly loose; in the special case with no channel extension (i.e. constant MIMO IC in Example 2.4a), this bound is weaker than the constant DoF bound (see [6-8] or Theorem 3.1). Nevertheless, it provides the first nontrivial DoF upper bound both for Example 2.4b (MIMO IC with constant channel extensions), and for Example 2.4c (MIMO IC with an arbitrary number generic extensions) with a general $K$.

\section{Algebraic Tools}


The zero-forcing conditions for interference alignment can be interpreted as a system of polynomial equations with the beamforming matrices being the variables. In this section, we present some technical tools from algebraic geometry related to the solvability of polynomial systems.

\subsection{Review of Field Theory and a Useful Lemma}

The goal of this subsection is to prove Lemma 4.1 that determines the infeasibility of a class of polynomial systems. Lemma 4.1 will be used in the proof of Theorem 3.2 in Section 6. To derive this Lemma, we first review the theory of transcendence degree (see Chapter 1 and Chapter 8 of [18] and [7]).

Let $\Omega / F$ be a field extension, i.e. $F$ and $\Omega$ are two fields such that $F \subseteq \Omega$. Denote $F\left[x_{1}, \ldots, x_{n}\right]$ as the polynomial ring which consists of all polynomials in variables $x_{1}, \ldots, x_{n}$ with coefficients in $F$. We say $\alpha_{1}, \ldots, \alpha_{n} \in \Omega$ are algebraically independent over $F$ if the only polynomial $P$ in $F\left[x_{1}, \ldots, x_{n}\right]$ that satisfies $P\left(\alpha_{1}, \ldots, \alpha_{n}\right)=0$ is $P=0$. Otherwise, we say $\alpha_{1}, \ldots, \alpha_{n}$ are algebraically dependent (i.e. there exists a nonzero polynomial $P$ such that $\left.P\left(\alpha_{1}, \ldots, \alpha_{n}\right)=0\right)$. The notion of algebraically independence/dependence is analogous to linear independence/dependence in linear algebra.

Example 4.1: Consider the field extension $\mathbb{R} / \mathbb{Q}$, where $\mathbb{R}$ is the field of real numbers, and $\mathbb{Q}$ is the field of rational numbers. For any rational number $q, \pi$ and $q$ are algebraically independent over $\mathbb{Q}$ since $\pi$ is not the root of any polynomial with rational coefficients. On the other hand, $\pi$ and $2 \sqrt{\pi}+1$ are algebraically dependent over $\mathbb{Q}$ because $P(\pi, 2 \sqrt{\pi}+1)=0$, where $P\left(z_{1}, z_{2}\right)=4 z_{1}-\left(z_{2}-1\right)^{2}$.

Example 4.2: Consider the field extension $\mathbb{C}\left(x_{1}, x_{2}\right) / \mathbb{C}$, where $\mathbb{C}\left(x_{1}, x_{2}\right)$ is the field of rational functions in variables $\left(x_{1}, x_{2}\right)$ with complex coefficients. A rational function has the form $f_{1} / f_{2}$, where $f_{1}, f_{2}$ are polynomials and $f_{2} \neq 0$. Note that the field of rational functions $\mathbb{C}\left(x_{1}, x_{2}\right)$ is different from the ring of polynomials $\mathbb{C}\left[x_{1}, x_{2}\right]$. The three rational functions

$$
g_{1}\left(x_{1}, x_{2}\right)=\frac{x_{2}^{2}}{x_{1}+1}, \quad g_{2}\left(x_{1}, x_{2}\right)=x_{1}, \quad g_{3}\left(x_{1}, x_{2}\right)=x_{1} x_{2}
$$

are algebraically dependent over $\mathbb{C}$. This is because $P\left(g_{1}, g_{2}, g_{3}\right)=0$ where $P\left(z_{1}, z_{2}, z_{3}\right)=z_{1} z_{2}^{2}\left(z_{2}+\right.$ 1) $-z_{3}^{2}$.

We say $\alpha \in \Omega$ is algebraically dependent on $A \subseteq \Omega$ if there exists $\beta_{1}, \ldots, \beta_{m} \in A$ and a polynomial $P\left(x_{1}, \ldots, x_{m}, y\right)$ such that $P\left(\beta_{1}, \ldots, \beta_{m}, \alpha\right)=0$ (i.e. $\beta_{1}, \ldots, \beta_{m}, \alpha$ are algebraically dependent) and $P\left(\beta_{1}, \ldots, \beta_{m}, y\right)$ is not a zero polynomial in the variable $y$. We say a set $B$ is algebraically dependent on $A$ if every element of $B$ is algebraically dependent on $A$. Note that the statement " $B$ is algebraically dependent on $A$ " is analogous to " $B$ can be spanned by $A$ " in linear algebra.

In linear algebra, a basis of a linear space is defined as a minimum set of vectors that can span all vectors in the linear space. In field theory, the transcendence basis is defined as follows: we say $A$ is a transcendental basis for the filed extension $\Omega / F$ if $A \in \Omega$ is an algebraically independent set such that $\Omega$ is algebraically dependent on $A$. The two definitions are both consistent with the notion of 
"minimum spanning set": a "basis" should be a minimum set of elements that can "span" all elements.

It can be shown that the transcendence basis exists and any two transcendence bases have the same cardinality (possibly infinite). Based on these results, we can define the transcendence degree of $\Omega / F$ as the cardinality of a transcendence basis for $\Omega / F$.

Example 4.3: Denote $\mathbb{C}\left(x_{1}, \ldots, x_{n}\right)$ as the field of rational functions in variables $x_{1}, \ldots, x_{n}$ with coefficients from $\mathbb{C}$. It can be shown that $g_{1}\left(x_{1}, \ldots, x_{n}\right)=x_{1}, \ldots, g_{n}\left(x_{1}, \ldots, x_{n}\right)=x_{n}$ is a transcendence basis of $\mathbb{C}\left(x_{1}, \ldots, x_{n}\right) / \mathbb{C}$. Hence the transcendence degree of $\mathbb{C}\left(x_{1}, \ldots, x_{n}\right) / \mathbb{C}$ is $n$.

The correspondences between linear algebra and the theory of transcendence degree are summarized in the following table [18]:

\begin{tabular}{|l|l|}
\hline Linear algebra & Transcendence \\
\hline linearly independent & algebraically independent \\
\hline A can be spanned by B & A is algebraically dependent on B \\
\hline basis & transcendence basis \\
\hline dimension & transcendence degree \\
\hline
\end{tabular}

In linear algebra, it can be shown that any $n+1$ vectors in a $n$-dimensional linear space are linearly dependent. In field theory, a similar result holds: for a field extension $\Omega / F$ with transcendence degree $n$, any $n+1$ elements in $\Omega$ are algebraically dependent over $F$ (a simple proof is to use Theorem 8.5 in [18]). The result for the case that $F=\mathbb{C}, \Omega=\mathbb{C}\left(x_{1}, \ldots, x_{n}\right)$ is useful for our problem, and we restate it in the following proposition.

Proposition 4.1 Consider the field extension $\mathbb{C}\left(x_{1}, \ldots, x_{n}\right) / \mathbb{C}$. Any $n+1$ rational functions

$$
g_{1}\left(x_{1}, \ldots, x_{n}\right), \ldots, g_{n+1}\left(x_{1}, \ldots, x_{n}\right)
$$

are algebraically dependent, i.e. there exists a nonzero polynomial $P \in \mathbb{C}\left[z_{1}, \ldots, z_{n+1}\right]$ such that $P\left(g_{1}, \ldots, g_{n+1}\right)=0$, or equivalently, $P\left(g_{1}\left(x_{1}, \ldots, x_{n}\right), \ldots, g_{n+1}\left(x_{1}, \ldots, x_{n}\right)\right)=0, \forall x_{1}, \ldots, x_{n}$.

In Example 6.2, we have shown that the three rational functions $g_{1}, g_{2}, g_{3} \in \mathbb{C}\left(x_{1}, x_{2}\right)$ are algebraically dependent. Proposition 4.1 implies that any three rational functions in $x_{1}, x_{2}$ are algebraically dependent.

Proposition 4.1 is more general than Example 4 in Section III of [7], which states that any $n+1$ polynomial functions in $\mathbb{C}\left[x_{1}, \ldots, x_{n}\right]$ are algebraically dependent. Reference [7] used this fact to show that for a certain type of polynomial systems of equations, a necessary condition for the solvability is that the number of variables is no less than the number of equations. Using the more general result Proposition 4.1, we show below that the dimensionality counting argument works for a broader class of systems. 
Lemma 4.1 Consider a system of polynomial equations and inequalities

$$
\begin{aligned}
\alpha_{i} f_{i}(x)+g_{i}(x) & =0, \quad i=1, \ldots, m, \\
f_{i}(x) \neq 0, & i=1, \ldots, m,
\end{aligned}
$$

where $\alpha=\left(\alpha_{1}, \ldots, \alpha_{m}\right)$ is a parameter vector, $x=\left(x_{1}, \ldots, x_{n}\right)$ is the variable and $f_{i}, g_{i}, i=1, \ldots, m$ are polynomial functions of $x$. If for a positive measure of $\alpha$, the above polynomial system (8) has a solution $x$, then the number of equations cannot exceed the number of variables, i.e. $m \leq n$.

Remark: Lemma 4.1 can be stated in a slightly different way: for generic $\alpha$, a necessary condition for (8) to be solvable is $m \leq n$.

Besides the polynomial equations in (8a), Lemma 4.1 adds an requirement that the coefficients of $\alpha_{i}$ are nonzero. This requirement guarantees that $\alpha_{i}$ can be expressed as a rational function $-g_{i}(x) / f_{i}(x)$ if $x$ is a solution to (8) ((8b) ensures that these rational functions are well defined). In the special case of $f_{i}=1$ for all $i$, Lemma 4.1 reduces to the key result proved in [7]. The difference of Lemma 4.1 and the corresponding result in [7] only lies in how $\alpha_{i}$ is expressed: $\alpha_{i}$ equals a rational function in the former case and a polynomial function in the latter case. The proof of Lemma 4.1 is given below.

Proof of Lemma 4.1: We prove by contradiction. Assume the contrary that $m>n$. Denote $\mathcal{I}$ as the set of $\alpha$ such that (8) has a solution $x$. The assumption of Lemma 4.1 is that $\mathcal{I}$ has a positive measure. By Proposition 4.1, the $m$ rational functions $-g_{i}(x) / f_{i}(x), i=1, \ldots, m$, in $n$ variable $x_{1}, \ldots, x_{n}$ are algebraically dependent. Thus, there exists a nonzero polynomial $P \in \mathbb{C}\left[z_{1}, \ldots, z_{m}\right]$ such that

$$
P\left(-\frac{g_{1}(x)}{f_{1}(x)}, \ldots,-\frac{g_{m}(x)}{f_{m}(x)}\right)=0, \quad \forall x .
$$

We claim that

$$
P\left(\alpha_{1}, \ldots, \alpha_{m}\right)=0, \quad \forall \alpha \in \mathcal{I} \text {. }
$$

In fact, for any $\alpha \in \mathcal{I}$, there exists a solution $x$ to (8), i.e. $\alpha_{i} f_{i}(x)=-g_{i}(x)$ and $f_{i}(x) \neq 0, i=1, \ldots, m$. Thus for any $\alpha \in \mathcal{I}$, there exists an $x$ such that $\alpha_{i}=-g_{i}(x) / f_{i}(x), i=1, \ldots, m$. Applying (9), we have

$$
P\left(\alpha_{1}, \ldots, \alpha_{m}\right)=P\left(-\frac{g_{1}(x)}{f_{1}(x)}, \ldots,-\frac{g_{m}(x)}{f_{m}(x)}\right)=0 .
$$

Thus, $P\left(\alpha_{1}, \ldots, \alpha_{m}\right)=0$ for a positive measure of $\alpha$, implying that $P$ is a zero polynomial, which is a contradiction.

Q.E.D.

\subsection{Solvability of a System with Generic Coefficients}

In this subsection, we consider a more restricted class of polynomial systems in which all coefficients are generic (in Lemma 4.1 only one parameter in each equation is generic). The results presented in this subsection can be derived from either Lemma 4.1 or Bernstein's theorem. 
Consider a system of polynomial equations with $m$ equations and $n$ variables:

$$
\mathcal{P}: \quad f_{k}\left(x_{1}, \ldots, x_{n}\right)=0, \quad k=1, \ldots, m,
$$

where $f_{i}, i=1, \ldots, m$ are nonzero polynomials in variables $x_{1}, \ldots, x_{n}$. Define

$$
\mathbb{C}^{*} \triangleq \mathbb{C} \backslash\{0\} .
$$

The following lemma states that a generic overdetermined system has no strictly nonzero solution (i.e. solution in $\left.\left(\mathbb{C}^{*}\right)^{n}\right)$.

Lemma 4.2 Consider a system of polynomial equations $\mathcal{P}$ as in (11). If $m \geq n+1$ and the coefficients of all polynomials $f_{k}$ are generic, then $\mathcal{P}$ has no solution in $\left(\mathbb{C}^{*}\right)^{n}$.

Proof of Lemma 4.2 (by Bernstein's theorem): Since $m \geq n+1$, we can pick a subsystem of $\mathcal{P}$ with $n$ polynomial equations. According to Corollary A.1 in Appendix A (a corollary of Bernstein's theorem), this subsystem has only a finite number of solutions in $\left(\mathbb{C}^{*}\right)^{n}$. These finite number of solutions in $\left(\mathbb{C}^{*}\right)^{n}$ can not satisfy the remaining $(m-n)$ generic equations in $\mathcal{P}$, thus $\mathcal{P}$ has no solution in $\left(\mathbb{C}^{*}\right)^{n}$, which proves Lemma 4.2.

Another Proof of Lemma 4.2 (by Lemma 4.1): Each nonzero polynomial $f_{k}$ contains at least one nonzero monomial, thus $f_{k}=0$ can be written as $\alpha_{k} h_{k}(x)+g_{k}(x)=0$, where $\alpha_{k}$ is a generic coefficient, $h_{k}(x)$ is a nonzero monomial, and $g_{k}$ is a polynomial. Since $m \geq n+1$, by Lemma 4.1, $\alpha_{k} h_{k}(x)+g_{k}(x)=$ $0, h_{k}(x) \neq 0, k=1, \ldots, m$ has no solution for generic $\alpha_{k}$ 's. Note that $x \in\left(\mathbb{C}^{*}\right)^{n}$ implies that all monomials $h_{k}(x) \neq 0$, thus $\mathcal{P}: \alpha_{k} h_{k}(x)+g_{k}(x)=0, x \in\left(\mathbb{C}^{*}\right)^{n}, k=1, \ldots, m$ has no solution either. Q.E.D.

Remark: The proof of Lemma 4.2 by Bernstein's theorem has appeared in [6, Section VI], though it is not explicitly stated that the term "solution" to a polynomial system should be interpreted as a strictly non-zero solution in $\left(\mathbb{C}^{*}\right)^{n}$.

Lemma 4.2 only considers solutions in $\left(\mathbb{C}^{*}\right)^{n}$. In the following, we use Lemma 4.2 to derive a sufficient condition under which an overdetermined system has no solution in $\mathbb{C}^{n}$. Define the support of a vector $x=\left(x_{1}, \ldots, x_{n}\right) \in \mathbb{C}^{n}$ as the set $\left\{j \mid x_{j} \neq 0\right\}$. For any support $J$, define a truncated variable $x_{J}=\left(x_{j}\right)_{j \in J}$ and its complement variable $\bar{x}_{J}=\left(x_{i}\right)_{i \notin J}$. We define a "partial-system" $\mathcal{P}_{J}$ by restricting the system $\mathcal{P}$ to the support $J$ as follows. In the system $\mathcal{P}$ set the variables $x_{i}, i \notin J$ to be 0 , then the monomials with any variable drawn from $\left\{x_{i} \mid i \notin J\right\}$ vanish and we obtain a new system

$$
\begin{gathered}
\mathcal{P}_{J}: f_{k J}\left(x_{J}\right)=0, \quad k=1, \ldots, m, \\
\text { where } f_{k J}\left(x_{J}\right)=f_{k}\left(x_{J}, \bar{x}_{J}\right) \quad \text { with } \bar{x}_{J}=0, \quad k=1, \ldots, m .
\end{gathered}
$$

Note that the partial-system $\mathcal{P}_{J}$ is different from a subsystem $\mathcal{Q}$ which consists of a subset of equations: in (12), $\mathcal{P}_{J}$ is defined by restricting $\mathcal{P}$ to a subset of variables $J$. We define

$$
\left|\mathcal{P}_{J}\right| \triangleq \text { number of nonzero polynomials in the set }\left\{f_{k J}, k=1, \ldots, m\right\} \text {. }
$$


Now we are ready to show that a generic system $\mathcal{P}$ has no solution in $\mathbb{C}^{n}$ if every partial-system $\mathcal{P}_{J}$ (including $\mathcal{P}$ itself) is overdetermined.

Corollary 4.1 Consider a system of polynomial equations $\mathcal{P}$ as in (11). Suppose the coefficients of all polynomials $f_{k}$ are generic and

$$
\left|\mathcal{P}_{J}\right| \geq|J|+1, \quad \forall J \subseteq\{1, \ldots, n\}
$$

where $\mathcal{P}_{J}$ is defined in (12). Then the system $\mathcal{P}$ has no solution in $\mathbb{C}^{n}$.

Proof of Corollary 4.1: The system $\mathcal{P}$ has no solution in $\mathbb{C}^{n}$ is equivalent to: for any subset $J \in$ $\{1, \ldots, n\}, \mathcal{P}$ has no solution with support $J$, or equivalently, the partial-system $\mathcal{P}_{J}$ has no solution in $\left(\mathbb{C}^{*}\right)^{|J|}$. Since $\left|\mathcal{P}_{J}\right| \geq|J|+1$, by applying Lemma 4.2 to the system $\mathcal{P}_{J}$, we obtain that $\mathcal{P}_{J}$ has no solution in $\left(\mathbb{C}^{*}\right)^{|J|}$.

Q.E.D.

Remark 1: Corollary 4.1 will be used to prove Proposition 4.2, a known DoF bound for constant MIMO IC, in Section 4.3. It will not be used to prove Theorem 3.1, but the idea of considering the solutions with specific supports is crucial for the proof of Theorem 3.1.

Remark 2: The idea of considering solutions with all possible supports has also been used in other related studies such as [19, Lemma 5]; see more discussions in Appendix A.

Corollary 4.1 implies that a necessary condition for a generic system $\mathcal{P}$ to have a solution in $\mathbb{C}^{n}$ is:

$$
\text { There exists a subset } J \in\{1, \ldots, n\} \text { such that }\left|\mathcal{P}_{J}\right| \leq|J| \text {. }
$$

In contrast, Lemma 4.2 implies that a necessary condition for a generic system $\mathcal{P}$ to have a solution in $\left(\mathbb{C}^{*}\right)^{n}$ is

$$
|\mathcal{P}| \leq n
$$

We give an example to illustrate the subtle and yet important difference of "solutions in $\left(\mathbb{C}^{*}\right)^{n}$ " and "solutions in $\mathbb{C}^{n}$ ". This example also shows that $|\mathcal{P}| \leq n$ is not a necessary condition for the feasibility of $\mathcal{P}$.

Example 4.4 Consider the following system with $n=2$ variables and $m=3$ equations:

$$
\begin{gathered}
f_{1}\left(x_{1}, x_{2}\right)=a_{1} x_{1}^{2}+a_{2} x_{1}=0 \\
f_{2}\left(x_{1}, x_{2}\right)=b_{1} x_{1} x_{2}+b_{2} x_{1}=0 \\
f_{3}\left(x_{1}, x_{2}\right)=c_{1} x_{1} x_{2}^{3}+c_{2} x_{1}=0
\end{gathered}
$$

where $a_{i}, b_{i}, c_{i}, i=1,2$ are generic coefficients. Obviously, $\left(x_{1}, x_{2}\right)=\left(0, x_{2}\right)$ is a solution to (16) for any $x_{2}$; thus (16) has infinitely many solutions in $\mathbb{C}^{2}$. In other words, $m>n$ does not imply the infeasibility of a generic system. If we require $x_{1}, x_{2} \in \mathbb{C}^{*}$, then $\mathcal{P}$ has no solution, i.e. $m>n$ implies that no strictly non-zero solution exists. 


\subsection{Applications to the constant MIMO IC}

A polynomial system of equations is called proper if in every subsystem the number of equations does not exceed the number of variables (see [6]). Researchers have tried to identify IA systems for which improperness implies infeasibility, since for such systems the DoF upper bound can be obtained by a dimensionality counting argument. The constant MIMO IC represents one such IA system. We emphasize that improperness does not imply infeasibility in general; even for the simple case that the coefficients are generic, counterexamples exist (see Example 4.4). Therefore, the counting argument works only when the problem exhibits some special structure. Below we will explain how the special structure of the constant MIMO IC makes the counting argument work.

Proposition 4.2 Consider the IA condition for the constant MIMO IC in the single beam case:

$$
\begin{aligned}
& u_{k}^{H} H_{k j} v_{j}=0, \quad \forall 1 \leq k \neq j \leq K, \\
& u_{k}^{H} H_{k k} v_{k} \neq 0, \quad \forall k,
\end{aligned}
$$

where $H_{k j}$ is a $M_{r} \times M_{t}$ matrix with generic entries, and $v_{k} \in \mathbb{C}^{M_{t} \times 1}, u_{k} \in \mathbb{C}^{M_{r} \times 1}, k=1, \ldots, K$ are beamformers. A necessary condition for (17a) to have a solution $\left\{u_{k}, v_{k}\right\}_{k=1}^{K}$ is $K(K-1) \leq K\left(M_{t}+\right.$ $\left.M_{r}-2\right)$, i.e. $K \leq M_{t}+M_{r}-1$. Hence, the DoF in the single-beam case is no more than $M_{t}+M_{r}-1$.

Proposition 4.2 is a special case of Theorem 3.1, and also a special case of the results in [6-8].

We describe in high-level terms how the special structure makes the counting argument possible. Lemma 4.2 and Corollary 4.1 show that for a generic system $\mathcal{P}$,

$$
\begin{gathered}
\mathcal{P} \text { has a solution in } \mathbb{C}^{n} \Longrightarrow \exists J \in\{1, \ldots, n\} \text { such that }\left|\mathcal{P}_{J}\right| \leq|J| ; \\
\mathcal{P} \text { has a solution in }\left(\mathbb{C}^{*}\right)^{n} \Longrightarrow|\mathcal{P}| \leq n .
\end{gathered}
$$

To apply the counting argument, we need to prove

$$
\mathcal{P} \text { has a solution in } \mathbb{C}^{n} \Longrightarrow|\mathcal{P}| \leq n \text {. }
$$

There are two simple methods to prove (19) by (18). The first one is to prove the equivalence of LHS (Left-Hand-Side) of (18a) and (18b) using the special structure of the problem, i.e.

$$
\mathcal{P} \text { has a solution in } \mathbb{C}^{n} \Longleftrightarrow \mathcal{P} \text { has a solution in }\left(\mathbb{C}^{*}\right)^{n} \text {. }
$$

Then combining (20) and (18b) proves (19). The second method is to prove the equivalence of RHS (Right-Hand-Side) of (18a) and (18b) using the special structure of the problem, i.e.

$$
\exists J \in\{1, \ldots, n\} \text { such that }\left|\mathcal{P}_{J}\right| \leq|J| \Longleftrightarrow|\mathcal{P}| \leq n \text {. }
$$

Then combining (21) and (18a) proves (19). 
The first method was adopted in [6]; see the last paragraph of Section VI in [6] for a proof sketch. It should be noted that certain details were not explicitly stated in the proof. The proof relies on the following structureless property of the channel: the product of a generic matrix and a unitary basis matrix is still a generic matrix. This structureless property implies that a solution in $\mathbb{C}^{n}$ can be mapped to a solution in $\left(\mathbb{C}^{*}\right)^{n}$ via a unitary transformation without altering the genericity of the channel matrix. Thus there is no loss of generality in restricting the solutions to $\left(\mathbb{C}^{*}\right)^{n}$ as opposed to $\mathbb{C}^{n}$, which establishes (20). This argument can be formalized by a probability computation presented by the authors of [6] in private communication.

Below we provide a different proof that follows the second method. We will prove (21) by showing $\left|\mathcal{P}_{J}\right|=\left|\mathcal{P}_{\{1, \ldots, n\}}\right|, \forall J$, which is also a consequence of the structureless property of MIMO channel matrices.

A new proof of Proposition 4.2: First, notice that the nonzero solution to (17a) $u_{k} \neq 0, v_{k} \neq 0, \forall k$ will satisfy (17b) automatically since $H_{k k}$ is a matrix with generic entries. Therefore, (17) is solvable iff (17a) has a nonzero solution. We need to prove: if (17a) has a nonzero solution $u_{k} \in \mathbb{C}^{n} \backslash\{0\}, v_{j} \in$ $\mathbb{C}^{n} \backslash\{0\}, \forall k, j$, then $K(K-1) \leq K\left(M_{r}+M_{t}-2\right)$.

Suppose $u_{k p_{k}} \neq 0, v_{j q_{j}} \neq 0,1 \leq p_{k} \leq M_{r}, 1 \leq q_{j} \leq M_{t}, \forall k, j$. We can scale $u_{k}, v_{j}$ such that $u_{k p_{k}}=1, v_{j q_{j}}=1^{1}$ and $\left(u_{k}, v_{k}\right)_{1 \leq k \leq K}$ is still a solution to (17a). After scaling, (17a) becomes

$$
\mathcal{P}: 0=u_{k}^{H} H_{k j} v_{j}=\sum_{(p, q)} u_{k p}^{*} H_{k j}(p, q) v_{j q}=H_{k j}\left(p_{k}, q_{j}\right)+\sum_{(p, q) \neq\left(p_{k}, q_{j}\right)} u_{k p}^{*} H_{k j}(p, q) v_{q s}, \quad \forall k \neq j,
$$

where $H_{k j}(p, q)$ denotes the $(p, q)$ 'th entry of $H_{k j} . \mathcal{P}$ is a system of polynomial equations with generic coefficients, since the coefficients of different equations come from different channel matrices. The number of variables in $\mathcal{P}$ is $n=K\left(M_{r}+M_{t}-2\right)$ and the number of equations in $\mathcal{P}$ is $m=K(K-1)$.

Note that $\left|\mathcal{P}_{J}\right|=m$ for any support $J \subseteq\{1, \ldots, n\}$, since each equation has a nonzero constant term $H_{k j}\left(p_{k}, q_{j}\right)$ and will not become a trivial equation $0=0$ by restricting to any support $J$. According to Corollary 4.1, a necessary condition for $\mathcal{P}$ to have a solution in $\mathbb{C}^{n}$ is $\left|\mathcal{P}_{J}\right| \leq|J|$ for some $J$. Thus we have $m=\left|\mathcal{P}_{J}\right| \leq|J|$. Combining with the fact $|J| \leq n$, we obtain $m \leq n$, i.e. $K(K-1) \leq$ $K\left(M_{r}+M_{t}-2\right)$.

Q.E.D.

In the above proof, a generic channel coefficient is separated from other terms in the expression (22), which is possible due to the structureless of the channel (in fact, if $H_{k j}$ is diagonal, then the separated term $H_{k j}\left(p_{k}, q_{j}\right)$ will be zero if $p_{k} \neq q_{j}$, thus we may not be able to separate a generic channel coefficient). The expression (22) is the key step in the above proof. Interestingly, seperating a generic channel coefficient is also a critical step in the proofs of $[7,8]$, though they apply different algebraic tools. Specifically, the proof of [7] applies Lemma 4.1 to the expression (22) with $\alpha$ being

\footnotetext{
${ }^{1}$ In [7], the authors assume that $u_{k 1}=v_{j 1}=1, \forall k, j$. Such an assumption is valid when $H_{k j}$ is a full generic matrix, but one should use caution when applying this assumption to other problems. In particular, for diagonal channel matrices considered in Theorem 3.1, the assumption $u_{k 1}=v_{j 1}=1, \forall k, j$ is no longer valid. Here, we choose to present a more complicated assumption $u_{k p_{k}}=1, v_{j q_{j}}=1$ since it does not require any property of the channel matrices.
} 
$H_{k j}\left(p_{k}, q_{j}\right)$ 's and $f_{i}$ being 1 . A benefit of using expression (22), compared with the other way of utilizing the structureless property (i.e. multiplying a nonsingular matrix does not change genericity), is that it can be generalized to the multi-beam case (see $[7,8])$.

The above two methods for proving (19) are based on the structureless property of the channel, thus it is not surprising that they can not be simply applied to structured channels, even with generic extensions. We will explain the difficulty of extending them to the MIMO IC with generic channel extensions (see Section 5.1).

\section{Proof of Theorem 3.1}

This section is devoted to the proof of Theorem 3.1 for the SISO IC in Section 5.2. The proof of Theorem 3.1 for the MIMO IC is a similar to the proof for the SISO IC, and is given in Appendix B. The major difference is that we only consider the supports of $u_{k}, v_{k}$ in SISO IC, while in MIMO IC we consider both the supports and the "block-supports" of $u_{k}, v_{k}$.

\subsection{Preliminary Analysis}

Consider a SISO IC with $T$ generic channel extensions. The IA condition is

$$
\begin{aligned}
& u_{k}^{H} H_{k j} v_{j}=0, \quad \forall 1 \leq k \neq j \leq K, \\
& u_{k}^{H} H_{k k} v_{k} \neq 0, \quad \forall 1 \leq k \leq K,
\end{aligned}
$$

where $H_{k j}=\operatorname{diag}\left(h_{k j}(1), \ldots, h_{k j}(T)\right)$ is a $T \times T$ diagonal matrix with generic diagonal entries, and $v_{j} \in \mathbb{C}^{T \times 1}, u_{k} \in \mathbb{C}^{T \times 1}$ are the beamforming vectors. After a simple scaling of $\left\{u_{k}, v_{k}\right\}$ (i.e., setting one of the entries of these vectors to 1 ), (23a) becomes a system of polynomial equations $\mathcal{P}$ with $n=2 K(T-1)$ variables and $m=K(K-1)$ equations.

Unlike the generic (full) channel matrices in the constant MIMO IC, $H_{k j}$ 's here are generic diagonal matrices. Due to the lack of the structureless property, the two methods described in Section 4.3 cannot be directly applied to this problem. Specifically, the first method proves (20) by using the invariance of genericity under unitary transformation: the product of a generic matrix and a unitary matrix $\Theta$ is still generic. Note that the purpose of introducing $\Theta$ is to map a beamformer in $\left(\mathbb{C}^{*}\right)^{n}$ to $(\mathbb{C})^{n}$, thus $\Theta$ should be a full matrix. For the IA system (23), the invariance property does not hold for generic diagonal matrices: the product of a generic diagonal matrix and a unitary matrix $\Theta$ is no longer diagonal (unless $\Theta$ itself is diagonal, which is not the case here). Thus (20) does not hold for our problem, i.e. we can not assume that the solutions are in $\left(\mathbb{C}^{*}\right)^{n}$.

The second method ("a new proof of Proposition 4.2") cannot be directly applied for our problem due to the following reason. To prove (21) for constant MIMO IC, we use the fact that $u_{k}^{H} H_{k j} v_{j}$ does 
not become zero when $u_{k}, v_{j}$ are nonzero vectors and $H_{k j}$ is a full generic matrix. For the IA system (23), $u_{k}^{H} H_{k j} v_{j}$ may become zero even if $u_{k}, v_{j}$ are nonzero: simply setting $u_{k 1}=0, v_{j 2}=\cdots=v_{j T}=0$ makes $u_{k}^{H} H_{k j} v_{j}$ vanish for any diagonal matrix $H_{k j}$. Thus unlike the constant MIMO IC case, here we can make $\left|\mathcal{P}_{J}\right|$ smaller than $m=\left|\mathcal{P}_{\{1, \ldots, n\}}\right|$. Moreover, $\left|\mathcal{P}_{J}\right|$ can be made as small as 0 : setting $u_{k 1}=0, v_{j 2}=\cdots=v_{j T}=0, \forall k, j$ makes all $u_{k}^{H} H_{k j} v_{j}$ vanish, which means $\left|\mathcal{P}_{J}\right|=0<|J|$ for the corresponding support $J$ when $T \geq 3$ (when $T \geq 3$, the variable vector has $|J|=K(T-2)>0$ variables after scaling). This example disproves (21) for the IA system (23).

Another more serious challenge arises when we further explore the example

$$
u_{k}=(0,1, \ldots, 1), \quad v_{j}=(1,0, \ldots, 0), \quad \forall k, j .
$$

We notice that (24) satisfies the zero-forcing condition (23a) for any $K$, thus it seems that no meaningful DoF upper bound can be derived from (23a). One may argue that (24) is not a valid example since it does not satisfy the full rank condition (23b). This observation reveals an important fact that may have not been explicitly recognized before: for structured channels, the (nonzero) solution to the zeroforcing condition does not satisfy the full rank condition automatically. This fact poses a new challenge to our problem: we need to take the inequalities (23b) into account (in contrast, in the constant MIMO IC the full rank condition (17b) can be discarded). In other words, we need to consider a hybrid system of equations and inequalities, rather than just a system of equations. Unfortunately, the solvability of hybrid systems is not the focus of algebraic geometry, thus conventional algebraic geometry tools cannot be directly applied.

Our proof technique can be briefly described as follows. We develop an induction analysis that leverages the recursive structure of the IA system, which implicitly utilizes the inequalities (i.e. the full rank condition). The algebraic tool Lemma 4.2 is used to prove a crucial intermediate bound (67).

\subsection{Proof of Theorem 3.1 for a SISO IC}

We define the maximal $K$ as $f(T)$, i.e.

$$
f(T) \triangleq \max \left\{K \mid(23) \text { is solvable for a positive measure of }\left(h_{k j}(t)\right)_{1 \leq k, j \leq K, 1 \leq t \leq T}\right\} .
$$

When $T=0$, we define $f(0)=0$.

To prove part (a) of Theorem 3.1, we only need to prove the following bound:

$$
f(T) \leq 2 T-1, \quad \forall T \geq 1
$$

We will do so using an induction argument.

For the basis of the induction $(T=1)$, it is easy to show that $f(1) \leq 2 T-1=1$. In fact, any two nonzero 1-dimensional vectors (i.e. scalars) are linearly dependent, thus when $K \geq 2$ the IA condition (23) has no solution. 
Now suppose (26) holds for each positive integer that is smaller than $T$. We prove that (26) holds for $T$. Suppose $K$ satisfies that the IA condition (23) has a solution $\left\{\tilde{u}_{k}, \tilde{v}_{k}\right\}_{k=1, \ldots, K}$ for a positive measure of $\left(h_{k j}(t)\right)$. For a vector $x=\left(x_{1}, \ldots, x_{T}\right)^{T} \in \mathbb{C}^{T}$, denote the support of $x$ as

$$
\operatorname{supp}(x)=\left\{j \mid x_{j} \neq 0\right\} .
$$

Each $\left(h_{k j}(t)\right)$ corresponds to (at least) one collection of $\operatorname{supports}\left\{\operatorname{supp}\left(\tilde{u}_{k}\right), \operatorname{supp}\left(\tilde{v}_{k}\right)\right\}_{k=1, \ldots, K}$. Since there are finitely many possible collections of supports, it follows that there exists a positive measure of $\left(h_{k j}(t)\right)$ which corresponds to the same collection of supports $\left\{R_{k}, S_{k}\right\}_{k=1, \ldots, K}$, where $R_{k}, S_{k} \subseteq$ $\{1, \ldots, T\}$. Denote this set of $\left(h_{k j}(t)\right)$ as $\mathcal{H}$ which has a positive measure.

Define a set of transmitter-receiver pairs as

$$
\begin{aligned}
\Omega & \triangleq\left\{(k, j) \mid 1 \leq k \neq j \leq K, \operatorname{supp}\left(\tilde{u}_{k}\right) \cap \operatorname{supp}\left(\tilde{v}_{j}\right)=\emptyset\right\} \\
& =\left\{(k, j) \mid 1 \leq k \neq j \leq K, R_{k} \cap S_{j}=\emptyset\right\}
\end{aligned}
$$

The complement of $\Omega$ in the set $\{(k, j) \mid 1 \leq k \neq j \leq K\}$ is

$$
\Omega^{c}=\left\{(k, j) \mid 1 \leq k \neq j \leq K, R_{k} \cap S_{j} \neq \emptyset\right\} .
$$

Furthermore, we denote $a_{k}, b_{k}$ as the number of nonzero entries in $\tilde{u}_{k}, \tilde{v}_{k}$ respectively, i.e.

$$
a_{k} \triangleq\left|\operatorname{supp}\left(\tilde{u}_{k}\right)\right|=\left|R_{k}\right|, \quad b_{k} \triangleq\left|\operatorname{supp}\left(\tilde{v}_{k}\right)\right|=\left|S_{k}\right|, \quad k=1, \ldots, K .
$$

Since $\tilde{u}_{k} \neq 0, \tilde{v}_{k} \neq 0, \forall k$, it follows that $a_{k} \geq 1$ and $b_{k} \geq 1$.

We will bound $\left|\Omega^{c}\right|$ by Claim 4.2 and bound $|\Omega|$ by the induction hypothesis. We first provide an upper bound on $\left|\Omega^{c}\right|$. Fix $r_{k} \in R_{k}, s_{k} \in S_{k}, k=1, \ldots, K$. In the system of equations (23a), scale each $u_{k}$ by $1 / u_{k r_{k}}$ and each $v_{k}$ by $1 / v_{k s_{k}}$. Then we obtain a new system of polynomial equations, denoted as $\mathcal{P}$, with $K(K-1)$ equations and $2 K(T-1)$ variables.

For any $\left(h_{k j}(t)\right) \in \mathcal{H}$, the IA condition (23) has a solution $\left\{\tilde{u}_{k}, \tilde{v}_{k}\right\}_{k=1, \ldots, K}$ with supports

$$
\operatorname{supp}\left(\tilde{u}_{k}\right)=R_{k}, \operatorname{supp}\left(\tilde{v}_{k}\right)=S_{k}, \quad k=1, \ldots, K .
$$

Since $r_{k} \in R_{k}, s_{k} \in S_{k}$, we have $\tilde{u}_{k r_{k}} \neq 0, \tilde{v}_{k s_{k}} \neq 0$. Scale each $\tilde{u}_{k}$ by $1 / \tilde{u}_{k r_{k}}$ and each $\tilde{v}_{k}$ by $1 / \tilde{v}_{k s_{k}}$, we obtain a new set of beamformers $\left\{\tilde{u}_{k} / \tilde{u}_{k r_{k}}, \tilde{v}_{k} / \tilde{v}_{k s_{k}}\right\}_{k=1, \ldots, K}$, which is a solution to the system $\mathcal{P}$. For simplicity, we still denote the scaled version $\left\{\tilde{u}_{k} / \tilde{u}_{k r_{k}}, \tilde{v}_{k} / \tilde{v}_{k s_{k}}\right\}_{k=1, \ldots, K}$ as $\left\{\tilde{u}_{k}, \tilde{v}_{k}\right\}_{k=1, \ldots, K}$ (i.e. assume $\left.\tilde{u}_{k r_{k}}=1, \tilde{v}_{k s_{k}}=1, \forall k\right)$.

The beamforming vectors $\tilde{u}_{k}, \tilde{v}_{k}, k=1, \ldots, K$ can be concatenated to form a vector in $\mathbb{C}^{2 K(T-1)}$ (after discarding the $2 K$ entries $\tilde{u}_{k r_{k}}, \tilde{v}_{k s_{k}}, k=1, \ldots, K$ since they have been normalized to 1 ). Let $J$ be the support of this concatenated vector. Then $J$ is a fixed subset of $\{1, \ldots, 2 K(T-1)\}$ determined by $R_{k} \backslash\left\{r_{k}\right\}, S_{k} \backslash\left\{s_{k}\right\}, k=1, \ldots, K$. The size of $J$ is

$$
|J|=\sum_{k}\left(a_{k}-1\right)+\sum_{k}\left(b_{k}-1\right)=\sum_{k}\left(a_{k}+b_{k}\right)-2 K .
$$


Consider the system of equations $\mathcal{P}_{J}$, obtained by restricting $\mathcal{P}$ to the subset of variables indexed by $J$ (i.e. set $u_{k r}=0$ if $r \notin R_{k}$ and $v_{k s}=0$ if $s \notin S_{k}$ in $\mathcal{P}$. See (12) for a formal definition of $\mathcal{P}_{J}$ ). We claim that the number of nonzero polynomial equations in $\mathcal{P}_{J}$ is exactly $\left|\Omega^{c}\right|$, i.e.

$$
\left|\mathcal{P}_{J}\right|=\left|\Omega^{c}\right|
$$

Note that $u_{k}^{H} H_{k j} v_{j}=\sum_{t} u_{k t}^{*} h_{k j}(t) v_{j t}$. Then we have

$$
\begin{aligned}
& u_{k}^{H} H_{k j} v_{j}=0 \text { does not become a zero equation in } \mathcal{P}_{J} \\
& \Longleftrightarrow\left.\tilde{u}_{k}^{H} H_{k j} \tilde{v}_{j} \neq 0 \text { (since the support of }\left\{\tilde{u}_{k}, \tilde{v}_{k}\right\} \text { is } J\right) \\
& \Longleftrightarrow \quad \exists t \text { such that } \tilde{u}_{k t}^{*} h_{k j}(t) \tilde{v}_{j t} \neq 0 \\
& \Longleftrightarrow \quad \exists t \in \operatorname{supp}\left(\tilde{u}_{k}\right) \cap \operatorname{supp}\left(\tilde{v}_{j}\right) \\
& \Longleftrightarrow \quad \operatorname{supp}\left(\tilde{u}_{k}\right) \cap \operatorname{supp}\left(\tilde{v}_{j}\right) \neq \emptyset \\
& \Longleftrightarrow \quad(k, j) \in \Omega^{c} .
\end{aligned}
$$

Therefore, $\left|\Omega^{c}\right|$ equals the number of nonzero equations in $\mathcal{P}_{J}$, which proves (30).

We then prove the following bound on $\left|\mathcal{P}_{J}\right|$ by Claim 4.2:

$$
\left|\mathcal{P}_{J}\right| \leq|J| \text {. }
$$

Assume the contrary, that $\left|\mathcal{P}_{J}\right| \geq|J|+1$. According to Claim 4.2, the system $\mathcal{P}_{J}$ has no solution in $\left(\mathbb{C}^{*}\right)^{|J|}$ for generic $\left(h_{k j}(t)\right)$. This contradicts the fact that for a positive measure of $\left(h_{k j}(t)\right)$ (in $\left.\mathcal{H}\right)$, the system $\mathcal{P}_{J}$ has a solution $\left\{\tilde{u}_{k}, \tilde{v}_{k}\right\}_{k=1, \ldots, K}$ in $\left(\mathbb{C}^{*}\right)^{|J|}$. Thus (31) is proved. Plugging (29) and (30) into (31), we obtain an upper bound on $\left|\Omega^{c}\right|$ :

$$
\left|\Omega^{c}\right| \leq \sum_{k}\left(a_{k}+b_{k}\right)-2 K
$$

Next, we provide an upper bound on $|\Omega|$. Define

$$
\Omega_{k}=\{j \mid(k, j) \in \Omega\}=\left\{j \mid j \in\{1, \ldots, K\} \backslash\{k\}, \operatorname{supp}\left(\tilde{u}_{k}\right) \cap \operatorname{supp}\left(\tilde{v}_{j}\right)=\emptyset\right\} .
$$

Then $\Omega=\bigcup_{k=1, \ldots, K} \Omega_{k}$. We will prove the following bound on $\left|\Omega_{k}\right|$ :

$$
\left|\Omega_{k}\right| \leq f\left(T-a_{k}\right), \quad k=1, \ldots, K
$$

Without loss of generality, assume $\operatorname{supp}\left(\tilde{u}_{k}\right)=\left\{1,2, \ldots, a_{k}\right\}$. Since $\operatorname{supp}\left(\tilde{v}_{j}\right) \cap \operatorname{supp}\left(\tilde{u}_{k}\right)=\emptyset, \forall j \in \Omega_{k}$, we have

$$
\tilde{v}_{j 1}=\cdots=\tilde{v}_{j a_{k}}=0, \quad \forall j \in \Omega_{k} .
$$

Consider a restriction of the beamforming vectors and channel matrices to the (T- $\left.a_{k}\right)$ dimensional space. Specifically, we define

$$
\begin{array}{r}
x_{j}=\left(\tilde{u}_{j, a_{k}+1}, \ldots, \tilde{u}_{j T}\right), \quad y_{j}=\left(\tilde{v}_{j, a_{k}+1}, \ldots, \tilde{v}_{j T}\right), \quad \forall j \in \Omega_{k}, \\
\hat{H}_{i j}=\operatorname{diag}\left(h_{i j}\left(a_{k}+1\right), \ldots, h_{i j}(T)\right), \quad \forall i, j \in \Omega_{k} .
\end{array}
$$


It follows from (34) that $\tilde{u}_{i}^{H} H_{i j} \tilde{v}_{j}=x_{i}^{H} \hat{H}_{i j} y_{j}, \forall i, j \in \Omega_{k}$. Since $\left\{\tilde{u}_{k}, \tilde{v}_{k}\right\}_{k=1, \ldots, K}$ satisfies the IA condition (23), we have that $\left\{x_{k}, y_{k}\right\}_{k=1, \ldots, K}$ satisfies

$$
\begin{aligned}
& x_{i}^{H} \hat{H}_{i j} y_{j}=0, \quad \forall i \neq j \in \Omega_{k}, \\
& x_{j}^{H} \hat{H}_{j j} y_{j} \neq 0, \quad \forall j \in \Omega_{k} .
\end{aligned}
$$

Note that (35) is the IA condition for a SISO IC with $\left(T-a_{k}\right)$ channel extensions and $\left|\Omega_{k}\right|$ users. For a positive measure of $\left(h_{k j}(t)\right)$ (in $\left.\mathcal{H}\right),(35)$ has a solution $\left\{x_{k}, y_{k}\right\}_{k=1, \ldots, K}$. By the definition of $f(\cdot)$ in (25), we have $\left|\Omega_{k}\right| \leq f\left(T-a_{k}\right)$, which proves (33).

Since $a_{k} \geq 1$, it follows from the induction hypothesis that $f\left(T-a_{k}\right) \leq 2\left(T-a_{k}\right)-1$ when $a_{k}<T$. Since $f(0)=0$, we have $f\left(T-a_{k}\right)=2\left(T-a_{k}\right)$ when $a_{k}=T$. In summary, we have

$$
f\left(T-a_{k}\right) \leq 2\left(T-a_{k}\right), \quad k=1, \ldots, K
$$

Combining (33) and (36), we obtain

$$
\left|\Omega_{k}\right| \leq 2\left(T-a_{k}\right), \quad k=1, \ldots, K
$$

Summing up (37) for $k=1, \ldots, K$, we obtain $|\Omega| \leq \sum_{k} 2\left(T-a_{k}\right)$. Similarly, we can prove $|\Omega| \leq$ $\sum_{k} 2\left(T-b_{k}\right)$. Combining these two bounds on $|\Omega|$, we get

$$
|\Omega| \leq \frac{1}{2}\left(\sum_{k} 2\left(T-a_{k}\right)+\sum_{k} 2\left(T-b_{k}\right)\right)=2 K T-\sum_{k}\left(a_{k}+b_{k}\right) .
$$

Finally, combining the bounds on $|\Omega|$ and $\left|\Omega^{c}\right|$ (cf. (32) and (38)), we can obtain the following bound on $K$

$$
K(K-1)=\left|\Omega^{c}\right|+|\Omega| \leq \sum_{k}\left(a_{k}+b_{k}\right)-2 K+2 K T-\sum_{k}\left(a_{k}+b_{k}\right)
$$

which simplifies to $K-1 \leq-2+2 T$ or equivalently $K \leq 2 T-1$. Thus $f(T) \leq 2 T-1$ holds for $T$. This completes the induction step, so that (26) holds for any $T$, as desired.

\section{Proof of Theorem 3.2}

In this section, we will first analyze the difficulties of proving Theorem 3.2, and then present the formal proof for $L=2$ in Section 6.2. The proof of Theorem 3.2 for general $L$ is similar to the proof for $L=2$, and is given in Appendix $\mathrm{C}$.

\subsection{Preliminary Analysis}

Consider a $K$-user interference channel of diversity order $L$, with each channel matrix given by $H_{i j}=$ $\tau_{i j}^{1} A_{1}+\cdots+\tau_{i j}^{L} A_{L}$, where $A_{1}, A_{2}, \ldots, A_{L} \in \Psi \subseteq C^{N_{r} \times N_{t}}$ (see the definition of $\Psi$ in (1)). In the 
single-beam case (i.e., $d_{k}=1$ for each $k$ ), the IA condition (3) becomes

$$
\begin{aligned}
& u_{k}^{H} H_{k j} v_{j}=0, \quad \forall 1 \leq k \neq j \leq K, \\
& u_{k}^{H} H_{k k} v_{k} \neq 0, \quad \forall 1 \leq k \leq K,
\end{aligned}
$$

where $v_{j} \in \mathbb{C}^{N_{t} \times 1}, u_{k} \in \mathbb{C}^{N_{r} \times 1}$ are transmit and receive beamforming vectors respectively.

Due to the symmetry between the transmit and receive beamforming vectors in the IA condition (39), we can assume without loss of generality that $N_{t} \leq N_{r}$, which then implies

$$
N=\min \left\{N_{r}, N_{t}\right\}=N_{t}
$$

The proof of Theorem 3.1 can not be directly applied to prove Theorem 3.2. There are two difficulties in applying the induction analysis and Lemma 4.2. First, Lemma 4.2 only applies to a system with generic coefficients, whereas the coefficients of the system (39a) are not generic. To resolve this difficulty, we use Lemma 4.1 that determines the infeasibility of a system where each equation has the form that a generic parameter equals a rational function of variables.

The second difficulty has to do with the use of induction analysis. For the IA condition (39), we need to consider the case that a subset $S$ of $v_{j}$ 's lie in a $N^{\prime}$-dim linear space, where $N^{\prime}<N$. To this end, we can express $v_{j}$ as $v_{j}=D \bar{v}_{j}, j \in S$, where $D \in \mathbb{C}^{N \times N^{\prime}}, \bar{v}_{j} \in \mathbb{C}^{N^{\prime} \times 1}$. Then $u_{k}^{H} H_{k j} v_{j}=$ $u_{k}^{H} H_{k j} D \bar{v}_{j}=u_{k}^{H} \bar{H}_{k j} \bar{v}_{j}, \forall k, j \in S$, where the new channel matrix $\bar{H}_{k j}=H_{k j} D=\sum_{l} \tau_{k j}^{l} A_{l} D \in \mathbb{C}^{N_{r} \times N^{\prime}}$. At the first glance, it seems that $\left\{u_{j}, \bar{v}_{j}\right\}_{j \in S}$ satisfies a new IA condition with different building blocks $A_{l} D, l=1, \ldots, L$ and a lower ambient transmit dimension $N^{\prime}$. However, the coefficients $\left\{\tau_{k j}^{l}\right\}$ can not be viewed as generic since they are not independent of $D$, the basis matrix of $\operatorname{span}\left\{v_{j}, j \in S\right\}$ (recall that $v_{j}$ 's are design variables that depend on the channel coefficients $\left\{\tau_{k j}^{l}\right\}$ ). As a result, the induction hypothesis can not be applied to the lower dimensional IA problem. To resolve this difficulty, we use a "lifting" technique. Specifically, we show that the original IA condition (39) implies a "lifted" IA condition in a higher dimensional space. As will be seen, the induction analysis can be applied to the lifted IA condition.

\subsection{Proof of Theorem 3.2 for $L=2$}

The proof of Theorem 3.2 consists of two steps: first, we "lift" the IA condition to a higher dimensional IA condition; second, using Lemma 4.1, we prove the bound for the "lifted" IA condition by induction on $N$.

In this subsection, we prove Theorem 3.2 for the case $L=2$. The case of general $L$ can be treated similarly and is given in Appendix C. As mentioned in Section 6.1, without loss of generality we can assume (40). We can also assume $\tau_{i j}^{1}=1, \forall i, j$ since multiplying $H_{i j}$ by a nonzero scalar $1 / \tau_{i j}^{1}$ does not affect the IA condition (77). For simplicity, we further denote $\tau_{i j}^{2}$ as $\tau_{i j}$. Now the channel model 
becomes

$$
H_{i j}=A_{1}+\tau_{i j} A_{2} .
$$

Denote $f(N)$ as the maximal $K$ such that IA is feasible; more specifically,

$$
\begin{aligned}
f(N) \triangleq & \max \left\{K \mid \text { for a positive measure of }\left(\tau_{i j}\right),\right. \\
& \left.\quad \text { the DoF tuple }\left(d_{1}, \ldots, d_{K}\right)=(1,1, \ldots, 1) \text { is achievable via vector space IA }\right\} \\
& =\max \left\{K \mid \text { for a positive measure of }\left(\tau_{i j}\right), \exists\left\{v_{k}\right\}_{k=1, \ldots, K}, \text { s.t. IA condition (77) holds }\right\} .
\end{aligned}
$$

Note that $f(N)$ depends on the receive dimension $N_{r}$ and the building blocks $A_{1}, A_{2}$. What we need to prove is: for any $N_{r}$ and $A_{1}, A_{2} \in \Psi$ as defined in (1), we have

$$
f(N) \leq 2 N+\frac{N^{2}}{4}
$$

Bounding $f(N)$ directly by the IA condition is difficult. Instead, we consider a lifted IA condition:

$$
\begin{gathered}
\left(\begin{array}{c}
v_{k} \\
\tau_{k k} v_{k}
\end{array}\right) \Perp \operatorname{span}\left\{\left(\begin{array}{c}
v_{j} \\
\tau_{k j} v_{j}
\end{array}\right) \mid j \in\{1,2, \ldots, K\} \backslash\{k\}\right\}, \quad \forall 1 \leq k \leq K, \\
v_{k} \in \mathbb{C}^{N \times 1} \backslash\{0\}, \quad \forall 1 \leq k \leq K .
\end{gathered}
$$

Define $g(N)$ as the maximal $K$ for the lifted IA condition to hold; more specifically,

$$
g(N)=\max \left\{K \mid \text { for a positive measure of }\left(\tau_{i j}\right), \exists\left\{v_{k}\right\}_{k=1, \ldots, K} \text {, s.t. IA condition (43) holds }\right\} \text {. }
$$

Note that $g(N)$ does not depend on $N_{r}, A_{1}, A_{2}$. The following lemma shows that $f(N)$ is upper bounded by $g(N)$.

Lemma 6.1 For any $N_{r}, N$ and any $N_{r} \times N$ building blocks $A_{1}, A_{2} \in \Psi$ as defined in (1), we have

$$
f(N) \leq g(N)
$$

Proof of Lemma 6.1: We only need to prove: for any given $\left(\tau_{i j}\right)$, if $\left\{v_{k}\right\}_{k=1}^{K}$ satisfies the IA condition (77), then $\left\{v_{k}\right\}_{k=1}^{K}$ satisfies the lifted IA condition (43). We prove by contradiction. Assume $\left\{v_{k}\right\}_{k=1}^{K}$ does not satisfy (43), then the independence condition does not hold for some $k$, i.e.

$$
\exists \lambda_{1}, \ldots, \lambda_{K}, \text { s.t. } \quad v_{k}=\sum_{j \neq k} \lambda_{j} v_{j}, \quad \tau_{k k} v_{k}=\sum_{j \neq k} \lambda_{j} \tau_{k j} v_{j} .
$$

Left multiplying the $l$ th equation of (46) by $A_{l}, l=1,2$ and summing up yields

$$
H_{k k} v_{k}=\sum_{j \neq k} \lambda_{j} H_{k j} v_{j}
$$

where we have used the equation $H_{k j}=A_{1}+\tau_{k j} A_{2}$. This contradicts the assumption that $\left\{v_{k}\right\}$ satisfies the IA condition (77).

Q.E.D. 
According to Lemma 6.1, we only need to prove

$$
g(N) \leq 2 N+\frac{N^{2}}{4}
$$

We prove (47) by induction on $N$. For the basis of the induction, we consider the case $N=1$. We need to prove $K \leq 2 N+N^{2} / 4=2.25$ or equivalently $K \leq 2$. Assume $K \geq 3$ and for a positive measure of $\left(\tau_{k j}\right)$, the lifted IA condition (43) has a solution $\left\{v_{k}\right\}_{k=1}^{K}$. Since scaling each $v_{j}$ by a nonzero factor $1 / v_{j}$ does not affect the linear independence condition, we have

$$
\begin{aligned}
&\left(\begin{array}{c}
v_{k} \\
\tau_{k k} v_{k}
\end{array}\right) \Perp \operatorname{span}\left\{\left(\begin{array}{c}
v_{j} \\
\tau_{k j} v_{j}
\end{array}\right) \mid j \in\{1,2, \ldots, K\} \backslash\{k\}\right\}, \quad \text { for } k=1, \\
& \Longleftrightarrow\left(\begin{array}{c}
1 \\
\tau_{11}
\end{array}\right) \Perp \operatorname{span}\left\{\left(\begin{array}{c}
1 \\
\tau_{12}
\end{array}\right),\left(\begin{array}{c}
1 \\
\tau_{13}
\end{array}\right)\right\} .
\end{aligned}
$$

The linear independence relation (48) can not hold for a positive measure of $\left(\tau_{11}, \tau_{12}, \tau_{13}\right)$, a contradiction. Therefore, (47) holds for $N=1$.

Suppose (47) holds for $1, \ldots, N-1$. We will prove (47) holds for $N$. To this end, suppose $K$ satisfies that for a positive measure of $\left(\tau_{i j}\right), \exists v_{k} \in \mathbb{C}^{N \times 1}, k=1, \ldots, K$, such that the lifted IA condition (43) holds. Let us introduce the receive beamforming vector $u_{k}=\left(\begin{array}{l}u_{k}^{1} \\ u_{k}^{2}\end{array}\right) \in \mathbb{C}^{2 N \times 1}$, where $u_{k}^{1}, u_{k}^{2} \in \mathbb{C}^{N \times 1}$, and transform the lifted IA condition (43) to the following zero-forcing type IA condition:

$$
\begin{aligned}
& u_{k}^{H}\left(\begin{array}{c}
v_{j} \\
\tau_{k j} v_{j}
\end{array}\right)=0, \quad \Longleftrightarrow\left(u_{k}^{1}\right)^{H} v_{j}+\tau_{k j}\left(u_{k}^{2}\right)^{H} v_{j}=0, \quad \forall k \neq j, \\
& u_{k}^{H}\left(\begin{array}{c}
v_{k} \\
\tau_{k k} v_{k}
\end{array}\right) \neq 0, \quad \forall k .
\end{aligned}
$$

Define a set of transmitter-receiver pairs as

$$
\Omega=\left\{(k, j) \mid 1 \leq k \neq j \leq K,\left(u_{k}^{1}\right)^{H} v_{j}=\left(u_{k}^{2}\right)^{H} v_{j}=0\right\} .
$$

The complement of $\Omega$ with respect to $\{(k, j) \mid 1 \leq k \neq j \leq K\}$ is given by

$$
\Omega^{c}=\left\{(k, j) \mid 1 \leq k \neq j \leq K,\left(u_{k}^{2}\right)^{H} v_{j} \neq 0\right\} .
$$

In addition, define

$$
\Omega_{k}=\{j \mid(k, j) \in \Omega\}=\left\{j \mid j \in\{1, \ldots, K\} \backslash\{k\},\left(u_{k}^{1}\right)^{H} v_{j}=\left(u_{k}^{2}\right)^{H} v_{j}=0\right\} .
$$

Then $\Omega=\bigcup_{1 \leq k \leq K} \Omega_{k}$.

Notice that for a positive measure of $\left(\tau_{i j}\right)$, the lifted zero-forcing type IA condition (49) has a solution $\left\{u_{k}, v_{k}\right\}_{k=1, \ldots, K}$. In other words, each $\left(\tau_{i j}\right)$ corresponds to at least one solution $\left\{u_{k}, v_{k}\right\}_{k=1, \ldots, K}$ which defines a collection of sets $\left\{\Omega_{k}\right\}_{k=1, \ldots, K} \subseteq\{1, \ldots, K\}^{K}$. Since there are finitely many subsets of 
$\{1, \ldots, K\}^{K}$, we must have one collection of sets $\left\{\Omega_{k}\right\}_{k=1, \ldots, K}$ that corresponds to a positive measure of $\left(\tau_{i j}\right)$. Denote this set of $\left\{\tau_{i j}\right\}$ (which has a positive measure) as $\mathcal{H}_{0}$.

Consider the collection of sets $\left\{\Omega_{k}\right\}_{1 \leq k \leq K}$ that corresponds to $\left(\tau_{i j}\right) \in \mathcal{H}_{0}$. We will bound $|\Omega|$ by induction and bound $\left|\Omega^{c}\right|$ by Lemma 4.1. Specifically, to derive an upper bound on $|\Omega|$, we use the definition of $\Omega_{k}$ to obtain

$$
\mathcal{U}_{k} \triangleq \operatorname{span}\left\{u_{k}^{1}, u_{k}^{2}\right\} \perp \mathcal{V}_{k} \triangleq \operatorname{span}\left\{v_{j} \mid j \in \Omega_{k}\right\}, \quad \forall 1 \leq k \leq K,
$$

which implies

$$
\operatorname{dim}\left(\mathcal{V}_{k}\right)+\operatorname{dim}\left(\mathcal{U}_{k}\right) \leq N, \quad \forall 1 \leq k \leq K
$$

Since $u_{k}=\left(\begin{array}{l}u_{k}^{1} \\ u_{k}^{2}\end{array}\right)$ is a nonzero vector, $\operatorname{dim}\left(\mathcal{U}_{k}\right) \geq 1$. Then

$$
p_{k} \triangleq \operatorname{dim}\left(\mathcal{V}_{k}\right) \leq N-\operatorname{dim}\left(\mathcal{U}_{k}\right) \leq N-1
$$

Although all $\left(\tau_{i j}\right)$ in $\mathcal{H}_{0}$ correspond to the same collection of sets $\left\{\Omega_{k}\right\}_{k=1, \ldots, K}$, they may correspond to different sets of dimensions $\left\{p_{k}\right\}_{k=1, \ldots, K}$. Using a similar argument as before, we can show that there exist a positive measure of $\left(\tau_{i j}\right)$ in $\mathcal{H}_{0}$ which corresponds to the same set of dimensions $\left\{p_{k}\right\}_{k=1, \ldots, K}$. These $\left(\tau_{i j}\right)$ form a subset of $\mathcal{H}_{0}$, denoted as $\mathcal{H}$.

Consider the collection of dimensions $\left\{p_{k}\right\}_{k=1, \ldots, K}$ that corresponds to $\left(\tau_{i j}\right) \in \mathcal{H}$. We prove that

$$
\left|\Omega_{k}\right| \leq g\left(p_{k}\right) \leq 2 p_{k}+\frac{p_{k}^{2}}{4}, \quad \forall 1 \leq k \leq K .
$$

Let $D \in \mathbb{C}^{N \times p_{k}}$ be the basis matrix of $\mathcal{V}_{k}$, and suppose $v_{j}=D \bar{v}_{j}, \forall j \in \Omega_{k}$, where $\bar{v}_{j} \in \mathbb{C}^{p_{k}} \backslash\{0\}$. From the lifted IA condition (43), we have

$$
\begin{aligned}
& \left(\begin{array}{c}
v_{i} \\
\tau_{i i} v_{i}
\end{array}\right) \Perp \quad \operatorname{span}\left\{\left(\begin{array}{c}
v_{j} \\
\tau_{i j} v_{j}
\end{array}\right) \mid j \in \Omega_{k} \backslash\{i\}\right\}, \quad \forall i \in \Omega_{k}, \\
& \Longleftrightarrow\left(\begin{array}{c}
D \bar{v}_{i} \\
\tau_{i i} D \bar{v}_{i}
\end{array}\right) \Perp \quad \operatorname{span}\left\{\left(\begin{array}{c}
D \bar{v}_{j} \\
\tau_{i j} D \bar{v}_{j}
\end{array}\right) \mid j \in \Omega_{k} \backslash\{i\}\right\}, \quad \forall i \in \Omega_{k}, \\
& \Longrightarrow\left(\begin{array}{c}
\bar{v}_{i} \\
\tau_{i i} \bar{v}_{i}
\end{array}\right) \Perp \operatorname{span}\left\{\left(\begin{array}{c}
\bar{v}_{j} \\
\tau_{i j} \bar{v}_{j}
\end{array}\right) \mid j \in \Omega_{k} \backslash\{i\}\right\}, \quad \forall i \in \Omega_{k} .
\end{aligned}
$$

The last step can be proved by contradiction. If (52b) does not hold, then the LHS (left-hand side) of (52b) belongs to the space on the RHS (right-hand side) of (52b). Multiply both sides by $\left(\begin{array}{ll}D & 0 \\ 0 & D\end{array}\right)$, we obtain that the LHS of (52a) belongs to the space on the RHS of (52a), which contradicts (52a).

Now for a positive measure of $\left(\tau_{i j}\right)_{i, j \in \Omega_{k}}$, there exist $\bar{v}_{i} \in \mathbb{C}^{p_{k}} \backslash\{0\}, i \in \Omega_{k}$ that satisfy (52b). By the definition of $g(\cdot),\left|\Omega_{k}\right| \leq g\left(p_{k}\right)$. Using the induction hypothesis, $g\left(p_{k}\right) \leq 2 p_{k}+\frac{p_{k}^{2}}{4}$. Thus, (51) is proved. Summing up (51) for $k=1, \ldots, K$, we have

$$
|\Omega|=\sum_{k=1}^{K}\left|\Omega_{k}\right| \leq \sum_{k}\left(2 p_{k}+\frac{p_{k}^{2}}{4}\right) .
$$


Next, we provide an upper bound on $\left|\Omega^{c}\right|$. Equation (49a) implies that

$$
\tau_{k j}=-\frac{\left(u_{k}^{1}\right)^{H} v_{j}}{\left(u_{k}^{2}\right)^{H} v_{j}}, \quad \forall(k, j) \in \Omega^{c} .
$$

The system of equations (54) in variables $u_{i}^{1}, u_{i}^{2}, v_{i}, i=1, \ldots, K$ (parameterized by $\left(\tau_{k j}\right)$ ) has $\left|\Omega^{c}\right|$ equations.

We compute the number of free variables in $\left\{u_{k}, v_{k}\right\}_{1 \leq k \leq K}$. Since scaling $v_{j}$ does not affect the system of equations (54), we can scale each $v_{j}$ to make one of its entries to be 1 . Therefore, the number of variables in $\left\{v_{i}\right\}_{1 \leq i \leq K}$ is $K(N-1)$. To count the free variables in $u_{k}$, notice that the condition (6.2) implies that $\left(u_{k}^{1}\right)^{H} v_{j}=0$ for all $j \in \Omega_{k}$. Since $\operatorname{span}\left\{v_{j}: j \in \Omega_{k}\right\}$ has dimension $p_{k}$, it follows that $p_{k}$ entries of $u_{k}^{1}$ can be written as linear functions of the remaining $N-p_{k}$ entries of $u_{k}^{1}$ (with coefficients being the rational functions of $\left\{v_{j}: j \in \Omega_{k}\right\}$ ). Similarly, $p_{k}$ entries of $u_{k}^{2}$ can be represented as linear functions of the remaining $N-p_{k}$ entries of $u_{k}^{2}$, with coefficients being some rational functions of $\left\{v_{j}: j \in \Omega_{k}\right\}$. Substituting these linear functions into the right hand sides of (54) yields a new representation of each $\tau_{k j}$ as a rational function of the $2\left(N-p_{k}\right)$ free variables in $u_{k}^{1}, u_{k}^{2}$ as well as the $K(N-1)$ variables in $v_{j}$ 's. Because of the homogeneity of these rational functions over the $2\left(N-p_{k}\right)$ free entries of $u_{k}$, we can further scale $u_{k}$ to make one of these entries to be 1 . Thus, the number of free variables in $u_{k}^{1}, u_{k}^{2}$ is $2\left(N-p_{k}\right)-1$. In summary, the number of free variables in $\left\{u_{k}, v_{k}\right\}_{k=1}^{K}$ is

$$
K(N-1)+\sum_{k}\left(2\left(N-p_{k}\right)-1\right)=K(3 N-2)-2 \sum_{k} p_{k} .
$$

For a positive measure of $\left(\tau_{k j}\right)_{(k, j) \in \Omega^{c}}$, the rational system (54) has a solution $\left\{u_{k}^{1}, u_{k}^{2}, v_{k}\right\}_{1 \leq k \leq K}$ such that $\left(u_{k}^{2}\right)^{H} v_{j} \neq 0$. It follows from Lemma 4.1 that the number of equations should not exceed the number of variables, i.e.

$$
\left|\Omega^{c}\right| \leq K(3 N-2)-2 \sum_{k} p_{k}
$$

With the bounds on $|\Omega|$ and $\left|\Omega^{c}\right|$, we can now provide an bound on $K$. Summing up (55) and (53) yields

$$
K(K-1)=|\Omega|+\left|\Omega^{c}\right| \leq \sum_{k}\left(2 p_{k}+\frac{p_{k}^{2}}{4}\right)+K(3 N-2)-2 \sum_{k} p_{k}
$$

implying

$$
K(K-1) \leq K(3 N-2)+\sum_{k} \frac{p_{k}^{2}}{4} \quad \text { or equivalently } \quad K \leq 3 N-1+\frac{1}{K} \sum_{k} \frac{p_{k}^{2}}{4} .
$$

Thus, if $p_{k} \leq N-2, \forall k$, then (56) leads to

$$
K \leq 3 N-1+\frac{1}{K} \sum_{k} \frac{p_{k}^{2}}{4} \leq 3 N-1+\frac{(N-2)^{2}}{4}=2 N+\frac{N^{2}}{4}
$$

as desired.

It remains to consider the case $p_{k}=N-1$ for some $k$. We need to prove the following claim. 
Claim 6.1 If there exists $k$ such that $p_{k}=N-1$, then

$$
K \leq\left|\Omega_{k}\right|+2
$$

Proof of Claim 6.1: According to (50), $1 \leq \operatorname{dim}\left(\mathcal{U}_{k}\right) \leq N-p_{k}=1$, thus $\operatorname{dim}\left(\mathcal{U}_{k}\right)=1$. Then $u_{k}^{1}$ is parallel to $u_{k}^{2}$. Without loss of generality, we assume $u_{k}^{1}=\gamma u_{k}^{2}$; then $\mathcal{U}_{k}=\operatorname{span}\left\{u_{k}^{2}\right\}$.

According to the IA condition (49a), we have

$$
0=\left(u_{k}^{1}\right)^{H} v_{j}+\tau_{k j}\left(u_{k}^{2}\right)^{H} v_{j}=\left(u_{k}^{2}\right)^{H} v_{j}\left(\gamma+\tau_{k j}\right), \quad \forall j \in\{1, \ldots, K\} \backslash\{k\} .
$$

Since $\gamma$ can be equal to at most one $-\tau_{k j}$, we have that $\gamma+\tau_{k j} \neq 0$ for at least $K-2 j$ 's. Therefore,

$$
0=\left(u_{k}^{2}\right)^{H} v_{j} \Longleftrightarrow u_{k}^{2} \perp v_{j}
$$

holds for at least $(K-2) j$ 's. Hence, $\Omega_{k}=\left\{j \mid \mathcal{U}_{k} \perp v_{j}\right\}$ has at least $(K-2)$ elements, which proves (58).

Q.E.D.

To complete the induction step, suppose $p_{k}=N-1$. Using (58) and (51), we have

$$
K \leq\left|\Omega_{k}\right|+2 \leq \frac{p_{k}^{2}}{4}+2 p_{k}+2=\frac{(N-1)^{2}}{4}+2(N-1)+2<\frac{N^{2}}{4}+2 N
$$

as desired. Combining this with (57) yields $K \leq 2 N+\frac{N^{2}}{4}$, which further implies $g(N) \leq 2 N+\frac{N^{2}}{4}$ holds for $N$. This completes the induction step, so that (47) holds for any $N$. Finally, combining (47) and Lemma 6.1, we obtain $f(N) \leq g(N) \leq 2 N+\frac{N^{2}}{4}$.

Q.E.D.

\section{Appendix}

\section{A Bernstein's Theorem and Dimensionality Counting Argument}

In this appendix, we clarify the use of Bernstein's theorem in the IA context where it was first cited in [6] as follows. Suppose $f_{i}$ is a polynomial function with $m_{i}$ monomials, and $c_{i j}$ is the coefficient of the $j$ 'th monomial, $j \in\left\{1,2, \ldots, m_{i}\right\}$. The number of common solutions of polynomials $f_{i}, \forall i$ is simply the number of solutions of the system equations $f_{i}=0, \forall i$.

Bernstein's theorem [6, Theorem 4] Given $n$ polynomials $f_{1}, \ldots, f_{n} \in \mathbb{C}\left[x_{1}, \ldots, x_{n}\right]$ with common solutions in $\left(\mathbb{C}^{*}\right)^{n}$, let $P_{i}$ be the Newton polytope of $f_{i}$ in $\mathbb{R}^{n}$. For independent random coefficients $c_{i j}, \forall i \in\{1, \ldots, n\}$ and $\forall j \in\left\{1,2, \ldots, m_{i}\right\}$, the number of common solutions is exactly equal to the mixed volume of Newton polytopes, $M V\left(P_{1}, \ldots, P_{n}\right)$.

We will not define the Newton polytope $P_{i}$ and mixed volume $M V\left(P_{1}, \ldots, P_{n}\right)$ here; interested readers can refer to [6] or [20] for the definitions and more information. The definition of the mixed 
volume may be useful for the achievability of DoF, though the difficulty seems to lie in how to determine whether the mixed volume is nonzero [6] (see some related work in [21,22]); nevertheless, these are beyond the scope of this paper. For our purpose of deciding the infeasibility of IA systems, we only need to know that the mixed volume $M V\left(P_{1}, \ldots, P_{n}\right)$ is a finite number for any polynomials $f_{1}, \ldots, f_{n}$. In other words, we are interested in the following corollary of Bernstein's theorem.

Corollary A.1 A generic system of polynomial equations with $n$ equations and $n$ variables has a finite number of solutions in $\left(\mathbb{C}^{*}\right)^{n}$.

We emphasize that in the above Bernstein's theorem, the term "common solutions" should be interpreted as "common solutions in $\left(\mathbb{C}^{*}\right)^{n}$ ", that is, "strictly nonzero common solutions (i.e., with no zero entries)". In fact, the number of "common solutions in $\mathbb{C}^{n}$ " of a generic system can be infinite; see Example 4.4. Bernstein's theorem has been applied to IA problems in $[6,12,13,23]$ to derive performance bounds. For the original application in the constant MIMO IC [6], the distinction between solutions in $\mathbb{C}^{n}$ and $\left(\mathbb{C}^{*}\right)^{n}$ can be ignored due to the structureless property of the channel (see Section 4.3 for discussion). For other problems in $[12,13,23]$, this distinction cannot be ignored and the results in these references require the artificial assumption that the IA solutions are strictly nonzero, though this assumption is not explicitly stated in these references.

An important clarification regarding the use of Bernstein's theorem is: many versions of Bernstein's theorem in the algebraic geometry literature do not imply Corollary A.1, thus do not imply the results in $[6,12,13,23]$ (even under the additional assumption of restricting to $\left.\left(\mathbb{C}^{*}\right)^{n}\right)$ and Corollary A.1. In particular, [24, Theorem 1], [25, Theorem 1.1] and [26, Theorem 7.1.4] state that the number of isolated zeros in $\left(\mathbb{C}^{*}\right)^{n}$ of any system $\mathcal{P}$ (possibly non-generic) is upper bounded by $M V\left(P_{1}, \ldots, P_{n}\right)$, and the bound is exact for generic choices of the coefficients. Note that the number of "isolated" solutions in $\left(\mathbb{C}^{*}\right)^{n}$ is not equal to the number of solutions in $\left(\mathbb{C}^{*}\right)^{n}$. For example, the system $x_{1}+x_{2}=1,\left(x_{1}+x_{2}\right)^{2}=1$ has infinitely many strictly nonzero solutions $\left(x_{1}, x_{2}\right)=(t,-t), \forall t \neq 0$, but none of these solutions is "isolated". This example shows that a finite number of isolated solutions in $\left(\mathbb{C}^{*}\right)^{n}$ does not imply a finite number of solutions in $\left(\mathbb{C}^{*}\right)^{n}$. Therefore, the version of Bernstein's theorem involves "isolated roots" does not imply the desired result Corollary A.1. Another version of Bernstein's theorem in [20, p. $346,(5.4)]$ starts with "Given Laurent polynomials $f_{1}, \ldots, f_{n}$ over $\mathbb{C}$ with finitely many common zeros in $\left(\mathbb{C}^{*}\right)^{n}$ ", which seems to be making the assumption that the number of solutions in $\left(\mathbb{C}^{*}\right)^{n}$ is finite ${ }^{2}$. However, we are interested in the question whether the finiteness assumption holds, which is not answered by this version of Bernstein's theorem.

Because of these possible confusions, we recommend the following version of Bernstein's theorem.

\section{Another version of Bernstein's theorem [27, Theorem 3.1]}

Consider polynomials $f_{1}, \ldots, f_{n} \in \mathbb{C}\left[x_{1}, \ldots, x_{n}\right]$ with Newton polytopes $P_{1}, \ldots, P_{n}$ in $\mathbb{R}^{n}$ :

\footnotetext{
${ }^{2}$ To be more precise, the statement in [20, p. 346, (5.4)] can be understood in two different ways, where the second way is that the finiteness assumption is made only to the non-generic case. Though algebraic geometry experts may be able to tell which way is correct by resorting to the proof of Bernstein's theorem, a reader in IA field probably can not.
} 
(a) For generic choices of the coefficients in $f_{1}, \ldots, f_{n}$, the number of common solutions of $f_{1}=\cdots=$ $f_{n}=0$ in $\left(\mathbb{C}^{*}\right)^{n}$ equals the mixed volume $M V\left(P_{1}, \ldots, P_{n}\right)$.

(b) For a specific specialization of the coefficients, the number of common solutions in $\left(\mathbb{C}^{*}\right)^{n}$ is either infinity, or does not exceed $M V\left(P_{1}, \ldots, P_{n}\right)$.

One may ask whether Bernstein's theorem can be extended to consider the number of solutions in $\mathbb{C}^{n}$ as opposed to $\left(\mathbb{C}^{*}\right)^{n}$. This motivates Corollary 4.1 which provides conditions on when a generic overdetermined system has no solution. We briefly discuss the connection of Corollary 4.1 and prior art. The extension of Bernstein's theorem to $\mathbb{C}^{n}$ has been studied extensively; see $[24,25,28]$ and the references therein. However, these results usually consider the number of isolated solutions and/or assume that the system has a finite number of solutions in $\mathbb{C}^{n}$. For example, [24, Theorem 2] states that the number of isolated zeros of a generic system equals the stable mixed volume provided that the system has finitely many roots. For the question of whether the finiteness assumption holds for a generic square system (i.e. the same number of equations and variables), a simple sufficient condition is provided in [24, Lemma 5], and a necessary and sufficient condition is provided in [28, Lemma 3]. Nevertheless, these two results can not be directly applied to prove that a generic overdetermined system has no solution under the conditions of these results. One may argue that if a subsystem has a finite number of solutions in $\mathbb{C}^{n}$, then these solutions can not satisfy the remaining polynomial equations with generic coefficients, thus the system is not solvable. A simple counterexample to this argument is that $\left(x_{1}, x_{2}, x_{3}\right)=(0,0,1)$ satisfies a polynomial equation $a x_{1}+b x_{2} x_{3}+c x_{1} x_{3}=0$ for generic $a, b, c$. To make this argument work, we need to assume, again, that these finite number of solutions are in $\left(\mathbb{C}^{*}\right)^{n}$. Therefore, Corollary 4.1 can only be derived from Bernstein's theorem, not from the previous results [24, Lemma 5] and [28, Lemma 3].

\section{B Proof of Theorem 3.1: General Case (MIMO IC)}

In this subsection, we present the proof of Theorem 3.1. As mentioned before, the proof for the MIMO IC case is not much harder than the SISO IC case, and the major difference is that we only consider the supports of $u_{k}, v_{k}$ in SISO IC, while in MIMO IC we consider both the supports and the "blocksupports" of $u_{k}, v_{k}$.

In an $M_{t} \times M_{r}$ MIMO IC with $T$ channel extensions, the channel matrix

$$
H_{k j}=\operatorname{diag}\left(H_{k j}^{1}, \ldots, H_{k j}^{T}\right)
$$

is a $M_{r} T \times M_{t} T$ block diagonal matrix, where each block $H_{k j}^{l}=\left(H_{k j}^{l}(p, q)\right)$ is a $M_{r} \times M_{t}$ matrix. The IA condition is given as follows:

$$
\begin{aligned}
& u_{k}^{H} H_{k j} v_{j}=0, \quad \forall 1 \leq k \neq j \leq K, \\
& u_{k}^{H} H_{k k} v_{k} \neq 0, \quad \forall 1 \leq k \leq K,
\end{aligned}
$$


where $v_{j} \in \mathbb{C}^{M_{t} T \times 1}, u_{k} \in \mathbb{C}^{M_{r} T \times 1}$ are beamformers.

Fix $M_{t}, M_{r}$ and define $f(T)$ as

$f(T) \triangleq \max \left\{K \mid(59)\right.$ is solvable for a positive measure of $\left.\left(H_{k j}^{l}(p, q)\right)_{1 \leq k, j \leq K, 1 \leq l \leq T, 1 \leq p \leq M_{r}, 1 \leq q \leq M_{t}}\right\}$.

To prove part (a) of Theorem 3.1, we only need to prove the following bound:

$$
f(T) \leq\left(M_{t}+M_{r}\right) T-1, \quad \forall T \geq 1 .
$$

We will do so by using an induction analysis.

For the basis of the induction $(T=1), f(1) \leq M_{t}+M_{r}-1$ holds according to Proposition 4.2. Now suppose (61) holds for any positive integer that is smaller than $T$. We will prove (61) holds for $T$. Suppose $K$ satisfies that (59) has a solution $\left\{\tilde{u}_{k}, \tilde{v}_{k}\right\}_{k=1, \ldots, K}$ for a positive measure of $\left(H_{k j}^{l}(p, q)\right)$. Denote the support of a vector $x$ as

$$
\operatorname{supp}(x) \triangleq\left\{j \mid x_{j} \neq 0\right\}
$$

$\operatorname{Each}\left(H_{k j}^{l}(p, q)\right)$ corresponds to (at least) one collection of $\operatorname{supports}\left\{\operatorname{supp}\left(\tilde{u}_{k}\right), \operatorname{supp}\left(\tilde{v}_{k}\right)\right\}_{k=1, \ldots, K}$. Since there are finitely many possible choices for the collection of supports, it follows that there exist a positive measure of $\left(H_{k j}^{l}(p, q)\right)$ which corresponds to the same collection of supports $\left\{R_{k}, S_{k}\right\}_{k=1, \ldots, K}$, where $R_{k} \subseteq\left\{1, \ldots, M_{r} T\right\}, S_{k} \subseteq\left\{1, \ldots, M_{t} T\right\}$. Denote this set of $\left(H_{k j}^{l}(p, q)\right)$ (which has a positive measure) as $\mathcal{H}$. Denote $\left\{\tilde{u}_{k}, \tilde{v}_{k}\right\}_{k=1, \ldots, K}$ as the solution of (59) for some $\left(H_{k j}^{l}(p, q)\right) \in \mathcal{H}$.

We divide $\tilde{u}_{k}$ and $\tilde{v}_{k}$ into $T$ blocks: $\tilde{u}_{k}=\left[\begin{array}{c}\tilde{u}_{k}^{1} \\ \vdots \\ \tilde{u}_{k}^{T}\end{array}\right], v_{k}=\left[\begin{array}{c}\tilde{v}_{k}^{1} \\ \vdots \\ \tilde{v}_{k}^{T}\end{array}\right]$, where each block $\tilde{u}_{k}^{l}=\left[\begin{array}{c}\tilde{u}_{k 1}^{l} \\ \vdots \\ \tilde{u}_{k M_{r}}^{l}\end{array}\right] \in \mathbb{C}^{M_{r} \times 1}$, $v_{k}^{l}=\left[\begin{array}{c}\tilde{v}_{k 1}^{l} \\ \vdots \\ \tilde{v}_{k M_{t}}^{l}\end{array}\right] \in \mathbb{C}^{M_{t} \times 1}$. For a vector with $T$ blocks $x=\left[\begin{array}{c}x^{1} \\ \vdots \\ x^{T}\end{array}\right]$, define the block-support of $x$ as

$$
\mathrm{B}-\operatorname{supp}(x)=\left\{t \mid x^{t} \neq 0\right\}
$$

Obviously, the support of $x$ determines its block-support (the reverse is not true). Since each $\left(H_{k j}^{l}(p, q)\right) \in$ $\mathcal{H}$ corresponds to the same collection of supports $\left\{R_{k}, S_{k}\right\}=\left\{\operatorname{supp}\left(\tilde{u}_{k}\right), \operatorname{supp}\left(\tilde{v}_{k}\right)\right\},\left(H_{k j}^{l}(p, q)\right) \in \mathcal{H}$ also corresponds to the same collection of block-supports $\left\{\mathrm{B}-\operatorname{supp}\left(\tilde{u}_{k}\right), \mathrm{B}-\operatorname{supp}\left(\tilde{v}_{k}\right)\right\} \triangleq\left\{R_{k}^{\mathrm{b}}, S_{k}^{\mathrm{b}}\right\}$. Define $\Omega$ as

$$
\begin{aligned}
\Omega & \triangleq\left\{(k, j) \mid 1 \leq k \neq j \leq K, \mathrm{~B}-\operatorname{supp}\left(\tilde{u}_{k}\right) \cap \mathrm{B}-\operatorname{supp}\left(\tilde{v}_{j}\right)=\emptyset\right\} \\
& =\left\{(k, j) \mid 1 \leq k \neq j \leq K, R_{k}^{\mathrm{b}} \cap S_{j}^{\mathrm{b}}=\emptyset\right\} .
\end{aligned}
$$

The complement of $\Omega$ in $\{(k, j) \mid 1 \leq k \neq j \leq K\}$ is

$$
\Omega^{c}=\left\{(k, j) \mid 1 \leq k \neq j \leq K, R_{k}^{\mathrm{b}} \cap S_{j}^{\mathrm{b}} \neq \emptyset\right\} .
$$


Furthermore, we denote $a_{k}, b_{k}$ as the number of nonzero blocks of $\tilde{u}_{k}, \tilde{v}_{k}$ respectively, i.e.

$$
a_{k} \triangleq\left|\mathrm{B}-\operatorname{supp}\left(\tilde{u}_{k}\right)\right|=\left|R_{k}^{\mathrm{b}}\right|, \quad b_{k} \triangleq\left|\mathrm{B}-\operatorname{supp}\left(\tilde{v}_{k}\right)\right|=\left|S_{k}^{\mathrm{b}}\right|, \quad \forall k .
$$

Since $\tilde{u}_{k} \neq 0, \tilde{v}_{k} \neq 0, \forall k$, it follows that $a_{k} \geq 1$ and $b_{k} \geq 1$.

We will bound $\left|\Omega^{c}\right|$ by Lemma 4.2 and bound $|\Omega|$ by the induction hypothesis. We first provide an upper bound on $\left|\Omega^{c}\right|$. Since scaling does not affect the solutions of (59), we can scale each $\tilde{u}_{k}, \tilde{v}_{k}$ to make one entry of them to be one. For simplicity, we still denote the scaled version of $\left\{\tilde{u}_{k}, \tilde{v}_{k}\right\}_{k=1, \ldots, K}$ as $\left\{\tilde{u}_{k}, \tilde{v}_{k}\right\}_{k=1, \ldots, K}$. After scaling, (59a) becomes a new system of polynomial equations with $K(K-$ 1) equations and $K\left(M_{t} T+M_{r} T-2\right)$ variables, denoted as $\mathcal{P}$. Then the system $\mathcal{P}$ has a solution $\left\{\tilde{u}_{k}, \tilde{v}_{k}\right\}_{k=1, \ldots, K}$.

The beamforming vectors $\tilde{u}_{k}, \tilde{v}_{k}, k=1, \ldots, K$ can be concatenated to form a vector in $\mathbb{C}^{K\left(M_{t} T+M_{r} T-2\right)}$ (after discarding the $2 K$ one's that are generated by scaling). Let $J$ be the support of this concatenated vector. Then $J$ is a subset of $\left\{1, \ldots, K\left(M_{t} T+M_{r} T-2\right)\right\}$ determined by the supports of $\tilde{u}_{k}, \tilde{v}_{k}, k=1, \ldots, K$ (excluding the positions of the $2 K$ one's). Therefore, the size of $J$ is upper bounded as (in SISO case, the inequality becomes equality)

$$
\begin{aligned}
|J| & =\sum_{k}\left(\left|\operatorname{supp}\left(\tilde{u}_{k}\right)\right|+\left|\operatorname{supp}\left(\tilde{v}_{k}\right)\right|\right)-2 K \\
& \leq \sum_{k}\left(\left|\mathrm{~B}-\operatorname{supp}\left(\tilde{u}_{k}\right)\right| M_{r}+\left|\mathrm{B}-\operatorname{supp}\left(\tilde{v}_{k}\right)\right| M_{t}\right)-2 K \\
& =\sum_{k}\left(a_{k} M_{r}+b_{k} M_{t}\right)-2 K,
\end{aligned}
$$

where the last equality follows from (63).

Consider the system of equations $\mathcal{P}_{J}$, which is obtained by restricting $\mathcal{P}$ to the subset of variables $J$. We claim that the number of nonzero polynomial equations in $\mathcal{P}_{J}$ is exactly $\left|\Omega^{c}\right|$, i.e.

$$
\left|\mathcal{P}_{J}\right|=\left|\Omega^{c}\right|
$$

For any $k \neq j, u_{k}^{H} H_{k j} v_{j}=\sum_{l}\left(u_{k}^{l}\right)^{H} H_{k j}^{l} v_{j}^{l}=\sum_{l, p, q}\left(u_{k p}^{l}\right)^{*} H_{k l}^{l}(p, q) v_{j q}^{l}$. Then we have

$$
\begin{aligned}
& u_{k}^{H} H_{k j} v_{j}=0 \text { does not become a zero equation in } \mathcal{P}_{J} \\
& \Longleftrightarrow \exists l, p, q, \text { s.t. }\left(\tilde{u}_{k p}^{l}\right)^{*} H_{k l}^{l}(p, q) \tilde{v}_{j q}^{l} \neq 0 \text { (since the support of }\left\{\tilde{u}_{i}, \tilde{v}_{i}\right\} \text { is } J \text { ) } \\
& \Longleftrightarrow \quad \exists l, p, q, \text { s.t. } \tilde{u}_{k p}^{l} \neq 0, \tilde{v}_{j q}^{l} \neq 0 \\
& \Longleftrightarrow \quad \exists l \in \mathrm{B} \text {-supp }\left(\tilde{u}_{k}\right) \cap \mathrm{B}-\operatorname{supp}\left(\tilde{v}_{j}\right) \\
& \Longleftrightarrow \mathrm{B}-\operatorname{supp}\left(\tilde{u}_{k}\right) \cap \mathrm{B}-\operatorname{supp}\left(\tilde{v}_{j}\right) \neq \emptyset \\
& \Longleftrightarrow(k, j) \in \Omega^{c} .
\end{aligned}
$$

Therefore, $\left|\Omega^{c}\right|$ equals the number of nonzero equations in $\mathcal{P}_{J}$, which proves (65). 
Since $\mathcal{P}_{J}$ has a solution in $\left(\mathbb{C}^{*}\right)^{|J|}$ for a positive measure of $\left(H_{k j}^{l}(p, q)\right) \in \mathcal{H}$, by Lemma 4.2, we have

$$
\left|\mathcal{P}_{J}\right| \leq|J|
$$

Plugging (64) and (65) into (66), we obtain an upper bound on $\left|\Omega^{c}\right|$ :

$$
\left|\Omega^{c}\right| \leq \sum_{k}\left(a_{k} M_{r}+b_{k} M_{t}\right)-2 K
$$

Next, we provide an upper bound on $|\Omega|$. Define

$$
\Omega_{k}=\{j \mid(k, j) \in \Omega\}=\left\{j \mid j \in\{1, \ldots, K\} \backslash\{k\}, \mathrm{B}-\operatorname{supp}\left(\tilde{u}_{k}\right) \cap \mathrm{B}-\operatorname{supp}\left(\tilde{v}_{j}\right)=\emptyset\right\} .
$$

Then $\Omega=\bigcup_{k=1, \ldots, K}\left\{(k, j) \mid j \in \Omega_{k}\right\}$ and $|\Omega|=\sum_{k=1}^{K}\left|\Omega_{k}\right|$. We claim that

$$
\left|\Omega_{k}\right| \leq f\left(T-a_{k}\right), \quad k=1, \ldots, K
$$

Without loss of generality, assume B-supp $\left(\tilde{u}_{k}\right)=\left\{1,2, \ldots, a_{k}\right\}$. Since B-supp $\left(\tilde{v}_{j}\right) \cap \mathrm{B}-\operatorname{supp}\left(\tilde{u}_{k}\right)=$ $\emptyset, \forall j \in \Omega_{k}$, we have that the first $a_{k}$ blocks of $\tilde{v}_{j}$ are zero, i.e.

$$
\tilde{v}_{j}^{1}=\cdots=\tilde{v}_{j}^{a_{k}}=0, \quad \forall j \in \Omega_{k} .
$$

Consider a restriction of the beamformers and channel matrices to the lower dimensional space for $\left(T-a_{k}\right)$ channel uses. Specifically, define

$$
\begin{gathered}
x_{j}=\left[\begin{array}{c}
\tilde{u}_{j}^{a_{k}+1} \\
\vdots \\
\tilde{u}_{j}^{T}
\end{array}\right], \quad y_{j}=\left[\begin{array}{c}
\tilde{v}_{j}^{a_{k}+1} \\
\vdots \\
\tilde{v}_{j}^{T}
\end{array}\right], \quad \forall j \in \Omega_{k}, \\
\hat{H}_{i j}=\operatorname{diag}\left(H_{i j}^{a_{k}+1}, \ldots, H_{i j}^{T}\right), \quad \forall i, j \in \Omega_{k} .
\end{gathered}
$$

It follows from (69) that $\tilde{u}_{i}^{H} H_{i j} \tilde{v}_{j}=x_{i}^{H} \hat{H}_{i j} y_{j}, \forall i, j \in \Omega_{k}$. Since $\left\{\tilde{u}_{k}, \tilde{v}_{k}\right\}_{k=1, \ldots, K}$ satisfies the IA condition (59), we have that $\left\{x_{k}, y_{k}\right\}_{k=1, \ldots, K}$ satisfies

$$
\begin{aligned}
& x_{i}^{H} \hat{H}_{i j} y_{j}=0, \quad \forall i \neq j \in \Omega_{k}, \\
& x_{j}^{H} \hat{H}_{j j} y_{j} \neq 0, \quad \forall j \in \Omega_{k} .
\end{aligned}
$$

Note that (70) is the IA condition for a $M_{t} \times M_{r}$ MIMO IC with $\left(T-a_{k}\right)$ channel extensions and $\left|\Omega_{k}\right|$ users. For a positive measure of $\left(H_{k j}^{l}(p, q)\right)$ (in $\left.\mathcal{H}\right)$, (70) has a solution $\left\{x_{k}, y_{k}\right\}_{k=1, \ldots, K}$. By the definition of $f(\cdot)$ in (60), we have $\left|\Omega_{k}\right| \leq f\left(T-a_{k}\right)$, which proves (68).

Since $a_{k} \geq 1$, it follows from the induction hypothesis that $f\left(T-a_{k}\right) \leq\left(M_{r}+M_{t}\right)\left(T-a_{k}\right)-1$ when $a_{k}<T$. Since $f(0)=0$, we have $f\left(T-a_{k}\right)=0=\left(M_{r}+M_{t}\right)\left(T-a_{k}\right)$ when $a_{k}=T$. In summary, we have

$$
f\left(T-a_{k}\right) \leq\left(T-a_{k}\right)\left(M_{r}+M_{t}\right), \quad k=1, \ldots, K
$$


Combining (68) and (71), we obtain

$$
\left|\Omega_{k}\right| \leq\left(T-a_{k}\right)\left(M_{r}+M_{t}\right), \quad k=1, \ldots, K
$$

Similarly, we have

$$
\left|\Omega_{k}\right| \leq\left(T-b_{k}\right)\left(M_{r}+M_{t}\right), \quad k=1, \ldots, K .
$$

Multiplying (72) by $\frac{M_{r}}{M_{t}+M_{r}}$ and (73) by $\frac{M_{t}}{M_{t}+M_{r}}$, and summing up them for $k=1, \ldots, K$ yields

$$
\left|\Omega_{k}\right| \leq T\left(M_{r}+M_{t}\right)-\left(a_{k} M_{r}+b_{k} M_{t}\right), \quad k=1, \ldots, K .
$$

Summing up (74) for $k=1, \ldots, K$ and applying the relation $|\Omega|=\sum_{k=1}^{K}\left|\Omega_{k}\right|$, we obtain

$$
|\Omega| \leq K T\left(M_{r}+M_{t}\right)-\sum_{k}\left(a_{k} M_{r}+b_{k} M_{t}\right)
$$

Finally, combining the bounds of $|\Omega|$ and $\left|\Omega^{c}\right|$ (c.f. (67) and (75)), we obtain the following bound

$$
K(K-1)=\left|\Omega^{c}\right|+|\Omega| \leq \sum_{k}\left(a_{k} M_{r}+b_{k} M_{t}\right)-2 K+K T\left(M_{r}+M_{t}\right)-\sum_{k}\left(a_{k} M_{r}+b_{k} M_{t}\right),
$$

which simplifies to $K-1 \leq-2+T\left(M_{r}+M_{t}\right)$, or equivalently $K \leq T\left(M_{r}+M_{t}\right)-1$. Thus, $f(T) \leq$ $T\left(M_{r}+M_{t}\right)-1$ holds for T. This completes the induction step, so that (61) holds for any $T$, as desired.

\section{Proof of Theroem 3.2 for General $L$}

Similar to the proof of Theorem 3.2 for $L=2$ in Section 6.2, the proof for general $L$ also consists of two steps: first, we "lift" the IA condition to a higher dimensional IA condition; second, using Lemma 4.1, we prove the bound for the "lifted" IA condition by induction on $N$.

As mentioned in Section 6.1, without loss of generality we can assume (40). We first eliminate the receive beamforming vectors from the IA condition (39) to obtain

$$
\begin{gathered}
H_{k k} v_{k} \quad \Perp \quad \operatorname{span}_{j \in\{1,2, \ldots, K\} \backslash\{k\}}\left\{H_{k j} v_{j}\right\}, \quad \forall 1 \leq k \leq K, \\
H_{k k} v_{k} \in \mathbb{C}^{N_{r} \times 1} \backslash\{0\}, \quad \forall 1 \leq k \leq K,
\end{gathered}
$$

where the notation $\Perp$ signifies linear independence. The IA condition (76) is solvable iff the IA condition (39) is solvable. Furthermore, since the basis matrices $\left(A_{1}, \ldots, A_{L}\right) \in \Psi$ satisfy the condition (1) and $N_{r} \leq N$, it follows that $H_{k k}$ has full column rank. Thus the condition $H_{k k} v_{k} \neq 0$ can be equivalently stated as $v_{k} \neq 0$. Consequently, the IA condition (76) can be further simplified as

$$
\begin{array}{cc}
H_{k k} v_{k} \Perp & \operatorname{span}_{j \in\{1,2, \ldots, K\} \backslash\{k\}}\left\{H_{k j} v_{j}\right\}, \quad \forall 1 \leq k \leq K, \\
v_{k} \in & \mathbb{C}^{N \times 1} \backslash\{0\}, \quad \forall 1 \leq k \leq K .
\end{array}
$$


Denote $f(N)$ as the maximal $K$ such that IA is feasible; more specifically,

$f(N)=\max \left\{K \mid\right.$ for a positive measure of $\left(\tau_{i j}^{l}\right)_{1 \leq i, j \leq K, 1 \leq l \leq L}, \exists\left\{v_{k}\right\}_{1 \leq k \leq K}$, s.t. IA condition (77) holds $\}$.

Note that $f(N)$ depends on the receive dimension $N_{r}$ and the building blocks $A_{1}, \ldots, A_{L}$. Then what we need to prove is: for any $N_{r}$ and $A_{1}, \ldots, A_{L} \in \Psi$ as defined in (1), we have

$$
f(N) \leq L N+\frac{N^{2}}{4}
$$

Define a lifted IA condition

$$
\begin{aligned}
& \left(\begin{array}{c}
\tau_{k k}^{1} v_{k} \\
\vdots \\
\tau_{k k}^{L} v_{k}
\end{array}\right) \Perp \operatorname{span}\left\{\left(\begin{array}{c}
\tau_{k j}^{1} v_{j} \\
\vdots \\
\tau_{k j}^{L} v_{j}
\end{array}\right) \mid j \in\{1,2, \ldots, K\} \backslash\{k\}\right\}, \forall k, \\
& v_{k} \in \mathbb{C}^{N \times 1} \backslash\{0\}, \forall k .
\end{aligned}
$$

Then we define $g(N)$ as the maximal $K$ for the lifted IA condition (79) to hold; more specifically,

$$
g(N)=\max \left\{K \mid \text { for a positive measure of }\left(\tau_{i j}^{l}\right), \exists\left\{v_{k}\right\}_{1 \leq k \leq K} \text {, s.t. IA condition (79) holds }\right\} .
$$

Note that $g(N)$ does not depend on $N_{r}, A_{1}, \ldots, A_{L}$.

Lemma C.1 For any $N_{r}, N$ and any $N_{r} \times N$ building blocks $A_{1}, \ldots, A_{L} \in \Psi$ as defined in (1), we have

$$
f(N) \leq g(N)
$$

\section{Proof of Lemma C.1:}

We only need to prove: for any given $\left(\tau_{i j}^{l}\right)$, if $\left\{v_{k}\right\}_{k=1}^{K}$ satisfies the IA condition (77), then $\left\{v_{k}\right\}_{k=1}^{K}$ satisfies the lifted IA condition (79). We prove by contradiction. Assume a set of nonzero vectors $\left\{v_{k}\right\}_{k=1}^{K}$ satisfies the IA condition (77), but does not satisfy (79), then for some $k$ the linear independence condition does not hold, i.e.

$$
\begin{aligned}
\exists \lambda_{1}, \ldots, \lambda_{K}, \text { s.t. } \quad \tau_{k k}^{1} v_{k}= & \sum_{j \neq k} \lambda_{j} \tau_{k j}^{1} v_{j}, \\
& \vdots \\
\tau_{k k}^{L} v_{k} & =\sum_{j \neq k} \lambda_{j} \tau_{k j}^{L} v_{j} .
\end{aligned}
$$

Left multiplying the $l$ th equation of (81) by $A_{l}$ and summing up these $L$ equations, we obtain

$$
H_{k k} v_{k}=\sum_{j \neq k} \lambda_{j} H_{k j} v_{j}
$$

where we use the equation $H_{i j}=\tau_{i j}^{1} A_{1}+\cdots+\tau_{i j}^{L} A_{L}$. This contradicts the assumption that $\left\{v_{k}\right\}$ satisfies the IA condition (77). Q.E.D. 
Remark: It can be easily verified that $f(N)=g(N)$ for the $1 \times L$ SIMO IC with $N$ constant extensions.

According to Lemma C.1, we only need to prove

$$
g(N) \leq L N+\frac{N^{2}}{4}
$$

We prove (82) by induction on $N$. For the basis of the induction, we consider the case $N=1$. We need to prove $K \leq L N+\frac{N^{2}}{4}=L+\frac{1}{4}$ or equivalently $K \leq L$. Assume $K \geq L+1$ and for a positive measure of $\left(\tau_{i j}^{l}\right)$, the lifted IA condition (79) has a solution $\left\{v_{k}\right\}_{k=1}^{K}$. Since scaling each $v_{j}$ by a nonzero factor $1 / v_{j}$ does not affect the linear independence condition, we have

$$
\begin{aligned}
& \left(\begin{array}{c}
\tau_{k k}^{1} v_{k} \\
\vdots \\
\tau_{k k}^{L} v_{k}
\end{array}\right) \Perp \operatorname{span}\left\{\left(\begin{array}{c}
\tau_{k j}^{1} v_{j} \\
\vdots \\
\tau_{k j}^{L} v_{j}
\end{array}\right) \mid j \in\{1,2, \ldots, K\} \backslash\{k\}\right\}, \quad \text { for } k=1, \ldots, K . \\
\Longrightarrow & \left(\begin{array}{c}
\tau_{11}^{1} \\
\vdots \\
\tau_{11}^{L}
\end{array}\right) \Perp \operatorname{span}\left\{\left(\begin{array}{c}
\tau_{12}^{1} \\
\vdots \\
\tau_{12}^{L}
\end{array}\right), \ldots,\left(\begin{array}{c}
\tau_{1, L+1}^{1} \\
\vdots \\
\tau_{1, L+1}^{L}
\end{array}\right)\right\} .
\end{aligned}
$$

Since there are $L$ vectors in the righthand side of the above relation and the ambient dimension is $L$, this linearly independence relation (83) can not hold for a positive measure of $\left(\tau_{i j}^{l}\right)$, a contradiction. Therefore, (82) holds for $N=1$.

Suppose (82) holds for $1, \ldots, N-1$. We will prove that (82) holds for $N$. To this end, suppose $K$ satisfies that for a positive measure of $\left(\tau_{i j}^{l}\right), \exists v_{k} \in \mathbb{C}^{N \times 1}, k=1, \ldots, K$, such that the lifted IA condition (79) holds. Let us introduce the receive beamformer $u_{k}=\left(\begin{array}{c}u_{k}^{1} \\ \vdots \\ u_{k}^{L}\end{array}\right) \in \mathbb{C}^{L N \times 1}$, where $u_{k}^{1}, \ldots, u_{k}^{L} \in \mathbb{C}^{N \times 1}$, and transform the lifted IA condition (79) to the following zero-forcing type IA condition:

$$
\begin{aligned}
& u_{k}^{H}\left(\begin{array}{c}
\tau_{k j}^{1} v_{j} \\
\vdots \\
\tau_{k j}^{L} v_{j}
\end{array}\right)=0 \quad \Longleftrightarrow \tau_{k j}^{1}\left(u_{k}^{1}\right)^{H} v_{j}+\cdots+\tau_{k j}^{L}\left(u_{k}^{L}\right)^{H} v_{j}=0, \quad \forall k \neq j, \\
& u_{k}^{H}\left(\begin{array}{c}
\tau_{k k}^{1} v_{k} \\
\vdots \\
\tau_{k k}^{L} v_{k}
\end{array}\right) \neq 0, \quad \forall k .
\end{aligned}
$$

Define a set of transmitter-receiver pairs as

$$
\Omega=\left\{(k, j) \mid 1 \leq k \neq j \leq K,\left(u_{k}^{1}\right)^{H} v_{j}=\cdots=\left(u_{k}^{L}\right)^{H} v_{j}=0\right\} .
$$

The complement of $\Omega$ with respect to $\{(k, j): 1 \leq k \neq j \leq K\}$ is given by

$$
\Omega^{c}=\left\{(k, j) \mid 1 \leq k \neq j \leq K, \exists \text { l, s.t. }\left(u_{k}^{l}\right)^{H} v_{j} \neq 0\right\} .
$$


In addition, define

$$
\Omega_{k}=\{j \mid(k, j) \in \Omega\}=\left\{j \mid j \in\{1, \ldots, K\} \backslash\{k\},\left(u_{k}^{1}\right)^{H} v_{j}=\cdots=\left(u_{k}^{L}\right)^{H} v_{j}=0\right\} .
$$

Then $\Omega=\bigcup_{k=1, \ldots, K}\left\{(k, j) \mid j \in \Omega_{k}\right\}$ and $|\Omega|=\sum_{k=1}^{K}\left|\Omega_{k}\right|$.

Notice that for a positive measure of $\left(\tau_{i j}^{l}\right)$, there exists $\left\{u_{k}, v_{k}\right\}_{k=1, \ldots, K}$ that satisfies the lifted zeroforcing type IA condition (84). In other words, each $\left(\tau_{i j}^{l}\right)$ corresponds to at least one solution $\left\{u_{k}, v_{k}\right\}_{k=1, \ldots, K}$ which defines a collection of sets $\left\{\Omega_{k}\right\}_{k=1, \ldots, K} \subseteq\{1, \ldots, K\}^{K}$. There are finitely many subsets of $\{1, \ldots, K\}^{K}$, thus there exists one collection of sets $\left\{\Omega_{k}\right\}_{k=1, \ldots, K}$, such that a positive measure of $\left(\tau_{i j}^{l}\right)$ correspond to $\left\{\Omega_{k}\right\}_{k=1}^{K}$. Denote this set of $\left(\tau_{i j}^{l}\right)$ (which has a positive measure) as $\mathcal{H}_{0}$.

Consider the collection of sets $\left\{\Omega_{k}\right\}_{1 \leq k \leq K}$ that corresponds to $\left(\tau_{i j}^{l}\right) \in \mathcal{H}_{0}$. We will bound $|\Omega|$ by the induction hypothesis and bound $\left|\Omega^{c}\right|$ by Lemma 4.1. Specifically, to derive an upper bound on $|\Omega|$, we use the definition of $\Omega_{k}$ to obtain

$$
\mathcal{U}_{k} \triangleq \operatorname{span}\left\{u_{k}^{1}, \ldots, u_{k}^{L}\right\} \perp \mathcal{V}_{k} \triangleq \operatorname{span}\left\{v_{j} \mid j \in \Omega_{k}\right\}, \quad \forall 1 \leq k \leq K,
$$

which implies

$$
\operatorname{dim}\left(\mathcal{V}_{k}\right)+\operatorname{dim}\left(\mathcal{U}_{k}\right) \leq N, \quad \forall 1 \leq k \leq K
$$

Since $u_{k}=\left(\begin{array}{c}u_{k}^{1} \\ \vdots \\ u_{k}^{L}\end{array}\right)$ is a nonzero vector, $\operatorname{dim}\left(\mathcal{U}_{k}\right) \geq 1$. Then

$$
p_{k} \triangleq \operatorname{dim}\left(\mathcal{V}_{k}\right) \leq N-\operatorname{dim}\left(\mathcal{U}_{k}\right) \leq N-1, \quad \forall 1 \leq k \leq K
$$

Although all $\left(\tau_{i j}^{l}\right)$ in $\mathcal{H}_{0}$ correspond to the same collection of sets $\left\{\Omega_{k}\right\}_{k=1, \ldots, K}$, they may correspond to different sets of dimensions $\left\{p_{k}\right\}_{k=1, \ldots, K}$. Using a similar argument as before, we can show that there exist a positive measure of $\left(\tau_{i j}^{l}\right)$ in $\mathcal{H}_{0}$ which corresponds to the same set of dimensions $\left\{p_{k}\right\}_{k=1, \ldots, K}$. These $\left(\tau_{i j}^{l}\right)$ form a subset of $\mathcal{H}_{0}$, denoted as $\mathcal{H}$.

Consider the collection of dimensions $\left\{p_{k}\right\}_{k=1, \ldots, K}$ that corresponds to $\left(\tau_{i j}\right) \in \mathcal{H}$. We prove that

$$
\left|\Omega_{k}\right| \leq g\left(p_{k}\right) \leq L p_{k}+\frac{p_{k}^{2}}{4}, \quad \forall 1 \leq k \leq K
$$

Let $D \in \mathbb{C}^{N \times p_{k}}$ be the basis matrix of $\mathcal{V}_{k}$, and suppose $v_{j}=D \bar{v}_{j}, \forall j \in \Omega_{k}$, where $\bar{v}_{j} \in \mathbb{C}^{p_{k}} \backslash\{0\}$. 
From the lifted IA condition (79), we have

$$
\begin{aligned}
& \left(\begin{array}{c}
\tau_{i i}^{1} v_{i} \\
\vdots \\
\tau_{i i}^{L} v_{i}
\end{array}\right) \Perp \quad \text { span }\left\{\left(\begin{array}{c}
\tau_{i j}^{1} v_{j} \\
\vdots \\
\tau_{i j}^{L} v_{j}
\end{array}\right) \mid j \in \Omega_{k} \backslash\{i\}\right\}, \forall i \in \Omega_{k}, \\
\Longleftrightarrow & \left(\begin{array}{c}
\tau_{i i}^{1} D \bar{v}_{i} \\
\vdots \\
\tau_{i i}^{L} D \bar{v}_{i}
\end{array}\right) \Perp \operatorname{span}\left(\left(\begin{array}{c}
\tau_{i j}^{1} D \bar{v}_{j} \\
\vdots \\
\tau_{i j}^{L} D \bar{v}_{j}
\end{array}\right) \mid j \in \Omega_{k} \backslash\{i\}\right\}, \forall i \in \Omega_{k}, \\
\Longrightarrow & \left(\begin{array}{c}
\tau_{i i}^{1} \bar{v}_{i} \\
\vdots \\
\tau_{i i}^{L} \bar{v}_{i}
\end{array}\right) \Perp \operatorname{span}\left\{\left(\begin{array}{c}
\tau_{i j}^{1} \bar{v}_{j} \\
\vdots \\
\tau_{i j}^{L} \bar{v}_{j}
\end{array}\right) \mid j \in \Omega_{k} \backslash\{i\}\right\}, \forall i \in \Omega_{k} .
\end{aligned}
$$

The last step can be proved by contradiction. If (88b) does not hold, then the LHS (left-hand side) of (88b) belongs to the space on the RHS (right-hand side) of (88b). Multiply both sides by $\left(\begin{array}{cccc}D & 0 & \cdots & 0 \\ 0 & D & \cdots & 0 \\ \vdots & \vdots & \ddots & \vdots \\ 0 & 0 & \cdots & D\end{array}\right) \in \mathbb{C}^{L N \times L p_{k}}$, we obtain that the LHS of (88a) belongs to the space on the RHS of (88a), which contradicts (88a).

Now for a positive measure of $\left(\tau_{i j}^{l}\right)_{i, j \in \Omega_{k}, 1 \leq l \leq L}$, there exist $\bar{v}_{i} \in \mathbb{C}^{p_{k}} \backslash\{0\}, i \in \Omega_{k}$ that satisfy (88b). By the definition of $g(\cdot),\left|\Omega_{k}\right| \leq g\left(p_{k}\right)$. Using the induction hypothesis, we have $g\left(p_{k}\right) \leq L p_{k}+\frac{p_{k}^{2}}{4}$. Thus, (87) is proved.

Summing up (87) for $k=1, \ldots, K$, we have

$$
|\Omega|=\sum_{k=1}^{K}\left|\Omega_{k}\right| \leq \sum_{k}\left(L p_{k}+\frac{p_{k}^{2}}{4}\right) .
$$

Next, we provide an upper bound on $\left|\Omega^{c}\right|$. For each pair $(k, j) \in \Omega^{c}$, there exists $l_{k j} \in\{1, \ldots, L\}$ such that $u_{k l_{k j}}^{H} v_{j} \neq 0$. Then equation (84a) implies that

$$
\tau_{k j}^{l_{k j}}=-\frac{\sum_{l \neq l_{k j}} \tau_{k j}^{l} u_{k l}^{H} v_{j}}{u_{k l_{k j}}^{H} v_{j}}, \quad \forall(k, j) \in \Omega^{c} .
$$

The system of rational equations (90) in variables $u_{i}^{1}, \ldots, u_{i}^{L}, v_{i}, i=1, \ldots, K$ (parameterized by $\left(\tau_{k j}^{l}\right)$ ) has $\left|\Omega^{c}\right|$ equations.

We compute the number of free variables in $\left\{u_{k}, v_{k}\right\}_{1 \leq k \leq K}$. Since scaling $v_{j}$ does not affect the system of equations (90), we can scale each $v_{j}$ to make one entry of it to be 1 . Therefore, the number of variables in $\left\{v_{i}\right\}_{1 \leq i \leq K}$ is $K(N-1)$. To count the number of free variables in $\left\{u_{k}\right\}$, notice that the 
condition (85) implies that $u_{k}^{1}, \ldots, u_{k}^{L}$ lie in $\mathcal{V}_{k}^{\perp}$ (the orthogonal complement of a fixed $p_{k}$-dim space $\mathcal{V}_{k}$ ). Since $\operatorname{span}\left\{v_{j}: j \in \Omega_{k}\right\}$ has dimension $p_{k}$, it follows that for each $l \in\{1, \ldots, L\}, p_{k}$ entries of $u_{k}^{l}$ can be represented as linear functions of the remaining $N-p_{k}$ entries of $u_{k}^{l}$, with coefficients being some rational functions of $\left\{v_{j}: j \in \Omega_{k}\right\}$. Substituting these linear functions into the right hand sides of (90) yields a new representation of each $\tau_{k j}^{l_{k j}}$ as a rational function of the $L\left(N-p_{k}\right)$ free variables in $u_{k}^{l}, l=1, \ldots, L$ as well as the $K(N-1)$ variables in $v_{j}$ 's. Because of the homogeneity of these rational functions over the $L\left(N-p_{k}\right)$ free entries of $u_{k}$, we can further scale $u_{k}$ to make one of these entries to be 1 . Thus, the number of free variables in $u_{k}$ is $L\left(N-p_{k}\right)-1$. In summary, the number of free variables in $\left\{u_{k}, v_{k}\right\}_{k=1}^{K}$ is

$$
K(N-1)+\sum_{k}\left(L\left(N-p_{k}\right)-1\right)=K((L+1) N-2)-L \sum_{k} p_{k} .
$$

For a positive measure of $\left(\tau_{k j}^{l}\right)_{(k, j) \in \Omega^{c}, 1 \leq l \leq L}$, the rational system (90) has a solution $\left\{u_{k}, v_{k}\right\}_{1 \leq k \leq K}$ such that $u_{k k_{k j}}^{H} v_{j} \neq 0$. By Lemma 4.1, the number of equations should not exceed the number of variables (given in (91)), i.e.

$$
\left|\Omega^{c}\right| \leq K((L+1) N-2)-L \sum_{k} p_{k}
$$

With the bounds on $|\Omega|$ and $\left|\Omega^{c}\right|$, we can now provide an bound on $K$. Summing up (92) and (89) yields

$$
K(K-1)=|\Omega|+\left|\Omega^{c}\right| \leq \sum_{k}\left(L p_{k}+\frac{p_{k}^{2}}{4}\right)+K((L+1) N-2)-L \sum_{k} p_{k},
$$

implying

$$
K(K-1) \leq K((L+1) N-2)+\sum_{k} \frac{p_{k}^{2}}{4} \text { or equivalently } K \leq(L+1) N-1+\frac{1}{K} \sum_{k} \frac{p_{k}^{2}}{4} .
$$

Therefore, if $p_{k} \leq N-2, \forall k$, then (93) leads to

$$
K \leq(L+1) N-1+\frac{1}{K} \sum_{k} \frac{p_{k}^{2}}{4} \leq(L+1) N-1+\frac{(N-2)^{2}}{4}=L N+\frac{N^{2}}{4},
$$

as desired.

It remains to consider the case $p_{k}=N-1$ for some $k$. In this case we have the following claim.

Claim C.1 If there exists $k$ such that $p_{k}=N-1$, then

$$
K \leq\left|\Omega_{k}\right|+L
$$

Proof of Claim C.1: According to (86), $1 \leq \operatorname{dim}\left(\mathcal{U}_{k}\right) \leq N-p_{k}=1$, thus $\operatorname{dim}\left(\mathcal{U}_{k}\right)=1$. Then $u_{k}^{1}, \ldots, u_{k}^{L}$ are parallel. Without loss of generality, we assume $u_{k}^{L} \neq 0$; then $\mathcal{U}_{k}=\operatorname{span}\left\{u_{k}^{L}\right\}$. Suppose $u_{k}^{t}=\gamma_{t} u_{k}^{L}, t=1, \ldots, L-1$. 
According to the IA condition (84a), we have

$$
0=\sum_{l} u_{k l}^{H} v_{j}=\left(u_{k}^{L}\right)^{H} v_{j}\left(\gamma_{1} \tau_{k j}^{1}+\cdots+\gamma_{L-1} \tau_{k j}^{L-1}+\tau_{k j}^{L}\right), \forall j \in\{1, \ldots, K\} \backslash\{k\} .
$$

Let $\Omega_{k}^{c}$ be the complement of $\Omega_{k}$ with respect to $\{1, \ldots, K\} \backslash\{k\}$. By the definition of $\Omega_{k}^{c}, j \in$ $\Omega_{k}^{c} \Rightarrow\left(u_{k}^{l}\right)^{H} v_{j} \neq 0$ for some $l$, which implies $\left(u_{k}^{L}\right)^{H} v_{j} \neq 0$. Combing with (96), we obtain

$$
0=\gamma_{1} \tau_{k j}^{1}+\cdots+\gamma_{L-1} \tau_{k j}^{L-1}+\tau_{k j}^{L}, \forall j \in \Omega_{k}^{c} .
$$

Since $\left(\gamma_{1}, \ldots, \gamma_{L-1}, 1\right)$ can be orthogonal to at most $L-1$ (generic) vectors $\left(\tau_{k j}^{1}, \ldots, \tau_{k j}^{L}\right)$, we obtain that $\left|\Omega_{k}^{c}\right| \leq L-1$, which implies $K \leq\left|\Omega_{k}\right|+L$.

Q.E.D.

To complete the induction step, suppose $p_{k}=N-1$ for some $k$. Using (95) and (87), we have

$$
K \leq\left|\Omega_{k}\right|+L \leq L p_{k}+\frac{p_{k}^{2}}{4}+L=L(N-1)+\frac{(N-1)^{2}}{4}+L<L N+\frac{N^{2}}{4},
$$

as desired. Combining this with (94) yields $K \leq L N+\frac{N^{2}}{4}$, which further implies $g(N) \leq L N+\frac{N^{2}}{4}$ holds for $N$. This completes the induction step, so that (82) holds for any $N$. Finally, combining (82) and Lemma C.1, we obtain $f(N) \leq g(N) \leq L N+\frac{N^{2}}{4}$.

\section{Acknowledgement}

We would like to thank Guy Bresler and David Tse for many fruitful discussions related to the subject of this paper. We are also grateful to Syed Jafar for the valuable discussions regarding the use of Bernstein's theorem.

\section{References}

[1] M.A. Maddah-Ali, A.S. Motahari, and A.K. Khandani, "Communication over MIMO X channels: Interference alignment, decomposition, and performance analysis," IEEE Transactions on Information Theory, vol. 54, no. 8, pp. 34573470, Aug. 2008.

[2] V.R. Cadambe and S.A. Jafar, "Interference alignment and degrees of freedom of the K-user interference channel," IEEE Transactions on Information Theory, vol. 54, no. 8, pp. 3425-3441, 2008.

[3] A. Host-Madsen and A. Nosratinia, "The multiplexing gain of wireless networks," in International Symposium on Information Theory (ISIT), 2005, pp. 2065-2069.

[4] A. S. Motahari, S. O. Gharan, M.-A. Mohammad-Ali, and A. K. Khandani, "Real interference alignment: Exploiting the potential of single antenna systems," arXiv preprint arXiv:0908.2282, 2009.

[5] A. Ghasemi, A.S. Motahari, and A.K. Khandani, "Interference alignment for the K user MIMO interference channel," in IEEE International Symposium on Information Theory Proceedings (ISIT), Jun. 2010, pp. 360-364.

[6] C.M. Yetis, T Gou, S.A. Jafar, and A.H. Kayran, "On feasibility of interference alignment in MIMO interference networks," IEEE Transactions on Signal Processing, vol. 58, no. 9, pp. 4771-4782, Sep. 2010. 
[7] M. Razaviyayn, G. Lyubeznik, and Z.-Q. Luo, "On the degrees of freedom achievable through interference alignment in a MIMO interference channel," IEEE Transactions on Signal Processing, vol. 60, no. 2, pp. 812-821, Feb. 2012.

[8] G. Bresler, D. Cartwright, and D. Tse, "Settling the feasibility of interference alignment for the MIMO interference channel: the symmetric square case," arXiv preprint arXiv:1104.0888, 2011.

[9] M. Razaviyayn, M. Sanjabi, and Z.-Q. Luo, "Linear transceiver design for interference alignment: Complexity and computation,” IEEE Transactions on Information Theory, vol. 58, no. 5, pp. 2896-2910, 2012.

[10] G. Bresler and D. Tse, "3 user interference channel: Degrees of freedom as a function of channel diversity," in Annual Allerton Conference on Communication, Control, and Computing (Allerton), Oct. 2009, pp. 265-271.

[11] V. R. Cadambe, S. A. Jafar, and C. Wang, "Interference alignment with asymmetric complex signaling - Settling the Høst-Madsen-Nosratinia conjecture,” IEEE Transactions on Information Theory, vol. 56, no. 9, pp. 4552-4565, 2010.

[12] C. Shi, R.A. Berry, and M.L. Honig, "Interference alignment in multi-carrier interference networks," in IEEE International Symposium on Information Theory Proceedings (ISIT). IEEE, 2011, pp. 26-30.

[13] R. Brandt, P. Zetterberg, and M. Bengtsson, "Interference alignment over a combination of space and frequency," in IEEE International Conference on Communications Workshops (ICC), 2013, pp. 149-153.

[14] S.A. Jafar, "Exploiting channel correlations - simple interference alignment schemes with no CSIT," in IEEE Global Telecommunications Conference (GLOBECOM), Dec. 2010, pp. 1-5.

[15] T. Gou, C. Wang, and S.A. Jafar, "Aiming perfectly in the dark-blind interference alignment through staggered antenna switching," IEEE Transactions on Signal Processing, vol. 59, no. 6, pp. 2734-2744, Jun. 2011.

[16] T. Gou and S.A. Jafar, "Degrees of freedom of the K user MIMO interference channel," in Asilomar Conference on Signals, Systems and Computers, Oct. 2008, pp. 126-130.

[17] K. Gomadam, V.R. Cadambe, and S.A. Jafar, "Approaching the capacity of wireless networks through distributed interference alignment," in IEEE Global Telecommunications Conference (GLOBECOM), Dec. 2008, pp. 1-6.

[18] P. Morandi, Field and Galois theory, Springer, 1996.

[19] B. Sturmfels, Solving systems of polynomial equations, vol. 97, American Mathematical Society, 2002.

[20] D. A. Cox, J. B. Little, and D. O’Shea, Using algebraic geometry, Springer New York, 1998.

[21] Óscar González, Carlos Beltrán, and Ignacio Santamaría, “On the feasibility of interference alignment for the k-user mimo channel with constant coefficients," arXiv preprint arXiv:1202.0186, 2012.

[22] Ó González, I Santamaria, and C Beltrán, "Finding the number of feasible solutions for linear interference alignment problems," in Information Theory Proceedings (ISIT), 2013 IEEE International Symposium on. IEEE, 2013, pp. 384388.

[23] S. H. Song, X. Chen, and K. B. Letaief, "Achievable diversity gain of K-user interference channel," in IEEE International Conference on Communications (ICC), 2012, pp. 4197-4201.

[24] B. Huber and B. Sturmfels, "Bernstein's theorem in affine space," Discrete \& Computational Geometry, vol. 17, no. 2, pp. 137-141, 1997.

[25] T. Li and X. Wang, "The BKK root count in $\mathbb{C}^{n}, "$ Mathematics of Computation of the American Mathematical Society, vol. 65 , no. 216, pp. 1477-1484, 1996.

[26] A. Dickenstein and I.Z. Emiris, Solving polynomial equations, Springer, 2005.

[27] I.Z. Emiris and J.F. Canny, "Efficient incremental algorithms for the sparse resultant and the mixed volume," Journal of Symbolic Computation, vol. 20, no. 2, pp. 117-149, 1995.

[28] J. M. Rojas and X. Wang, "Counting affine roots of polynomial systems via pointed newton polytopes," Journal of Complexity, vol. 12, no. 2, pp. 116-133, 1996. 